\title{
THE MASSIVE HOSTS OF RADIO GALAXIES ACROSS COSMIC TIME
}

\author{
Nick Seymour, ${ }^{1}$ Daniel Stern, ${ }^{2}$ Carlos De Breuck, ${ }^{3}$ Joel Vernet, ${ }^{3}$ Alessandro Rettura, ${ }^{4}$ Mark Dickinson, ${ }^{5}$ \\ Arjun Dey, ${ }^{6}$ Peter Eisenhardt, ${ }^{2}$ Robert Fosbury, ${ }^{3}$ Mark Lacy, ${ }^{1}$ Pat McCarthy, ${ }^{6}$ George Miley, ${ }^{7}$ \\ Brigitte Rocca-Volmerange, ${ }^{8}$ Hudb Röttgering, ${ }^{9}$ S. Adam Stanford, ${ }^{10,11}$ Harry Teplitz, ${ }^{1}$ \\ Wil van BReugel, ${ }^{10,11}$ AND ANDREW Zirm ${ }^{4}$ \\ Received 2007 January 2; accepted 2007 March 2
}

\begin{abstract}
We present the results of a comprehensive Spitzer survey of 69 radio galaxies across $1<z<5.2$. Using IRAC $(3.6-8.0 \mu \mathrm{m})$, IRS $(16 \mu \mathrm{m})$, and MIPS $(24-160 \mu \mathrm{m})$ imaging, we decompose the rest-frame optical to infrared spectral energy distributions into stellar, AGN, and dust components and determine the contribution of host galaxy stellar emission at rest-frame $H$ band. Stellar masses derived from rest-frame near-IR data, where AGN and young star contributions are minimized, are significantly more reliable than those derived from rest-frame optical and UV data. We find that the fraction of emitted light at rest-frame $H$ band from stars is $>60 \%$ for $\sim 75 \%$ of the high-redshift radio galaxies. As expected from unified models of AGNs, the stellar fraction of the rest-frame $H$-band luminosity has no correlation with redshift, radio luminosity, or rest-frame mid-IR $(5 \mu \mathrm{m})$ luminosity. In addition, while the stellar $H$-band luminosity does not vary with stellar fraction, the total $H$-band luminosity anticorrelates with the stellar fraction as would be expected if the underlying hosts of these radio galaxies comprise a homogeneous population. The resultant stellar luminosities imply stellar masses of $10^{11}-10^{11.5} M_{\odot}$ even at the highest redshifts. Powerful radio galaxies tend to lie in a similar region of mid-IR color-color space as unobscured AGNs, despite the stellar contribution to their mid-IR SEDs at shorter wavelengths. The mid-IR luminosities alone classify most HzRGs as LIRGs or ULIRGs with even higher total-IR luminosities. As expected, these exceptionally high mid-IR luminosities are consistent with an obscured, highly accreting AGN. We find a weak correlation of stellar mass with radio luminosity.
\end{abstract}

Subject headings: galaxies: active — galaxies: evolution — galaxies: high-redshift

Online material: color figures

\section{INTRODUCTION}

Luminous radio galaxies were the first class of obscured, or type 2, quasars to be discovered and characterized. They have accreting supermassive black holes whose continuum emission at UV, optical, and soft X-ray energies is absorbed by dust, thus allowing a clear view of the host galaxy. More recently, hard $\mathrm{X}$-ray and mid-IR surveys have identified the radio-quiet cousins to luminous radio galaxies (e.g., Norman et al. 2002; Stern et al. 2002; Martínez-Sansigre et al. 2005; Polletta et al. 2006; Lacy et al. 2007). The main evidence that radio galaxies host supermassive black holes comes from the high luminosities of their radio lobes, which are fed by radio jets originating at the host galactic nuclei. The lobe spatial extents (up to a few Mpc; Saripalli et al. 2005) and luminosities $\left(L_{1.4 \mathrm{GHz}} \geq 10^{25} \mathrm{~W} \mathrm{~Hz}^{-1}\right)$ clearly rule out a stellar origin for their energetics (e.g., Rees 1978). In terms of the orientation unification scheme for active galactic

\footnotetext{
1 Spitzer Science Center, California Institute of Technology, 1200 East California Boulevard, Pasadena, CA 91125; seymour@ipac.caltech.edu.

2 Jet Propulsion Laboratory, California Institute of Technology, Pasadena, CA 91109.

3 European Southern Observatory, Karl Schwarzschild Straße, D-85748 Garching, Germany.

4 Johns Hopkins University, 3400 North Charles Street, Baltimore, MD 21218

5 National Optical Astronomy Observatory, Tucson, AZ 85719.

6 Carnegie Observatories, 813 Santa Barbara Street, Pasadena, CA 91101.

7 Leiden Observatory, University of Leiden, P.O. Box 9513, 2300 RA Leiden, Netherlands.

8 Institute d'Astrophysique de Paris, 98bis Bd Arago, 75014 Paris, France.

9 University of California, Davis, CA 95616.

${ }^{10}$ Institute of Geophysics and Planetary Physics, Lawrence Livermore National Laboratory, Livermore, CA 94551.

11 University of California, Merced, P.O. Box 2039, Merced, CA 95344.
}

nuclei (AGN) (e.g., Barthel 1989; Antonucci 1993; Urry \& Padovani 1995), radio galaxies are radio-loud quasars seen from an angle where an optically thick torus obscures emission from the region closest to the central engine.

Due to their large radio luminosities, radio galaxies were the predominant way to probe the distant universe until the advent of 8-10 m class telescopes and the Lyman-break technique in the last decade. In fact, radio galaxies were the first galaxies to be found above redshifts one, two, three, and four (see Stern \& Spinrad 1999 and references therein). Across cosmic time, the host galaxies of powerful radio sources appear to be uniquely robust indicators of the most massive galaxies in the universe. At low redshift, this result has been known since the first optical identifications of extragalactic radio sources showed them to be associated with massive, giant elliptical ( $\mathrm{gE}$ and $\mathrm{cD}$ ) galaxies (Matthews et al. 1964). In the more distant universe, indirect evidence that this association remains intact comes from the detection of host galaxies with $r^{1 / 4}$ law light profiles in Hubble Space Telescope (HST) observations of high-redshift radio galaxies (HzRGs) at $1 \lesssim z \lesssim 2$ (Pentericci et al. 2001; Waddington et al. 2002; Zirm et al. 2003; A. J. Bunker et al. 2007, in preparation); the tendency for HzRGs to reside in moderately rich (proto-) cluster environments (Le Fèvre et al. 1996; Pascarelle et al. 1996; Venemans et al. 2002, 2003, 2004, 2005, 2007; Stern et al. 2003); the spectacular $(>100 \mathrm{kpc}$ ) luminous Ly $\alpha$ halos seen around several sources, implying large gas reservoirs (Reuland et al. 2003; Villar-Martín et al. 2003); submillimeter detections of HzRGs, implying violent star formation activity up to $\sim 1000 M_{\odot} \mathrm{yr}^{-1}$ (Archibald et al. 2001; Reuland et al. 2004); and a few direct kinematic measurements of HzRGs (Dey et al. 1996; Dey \& Spinrad 1996; Nesvadba et al. 2006). The most compelling evidence of this association of 


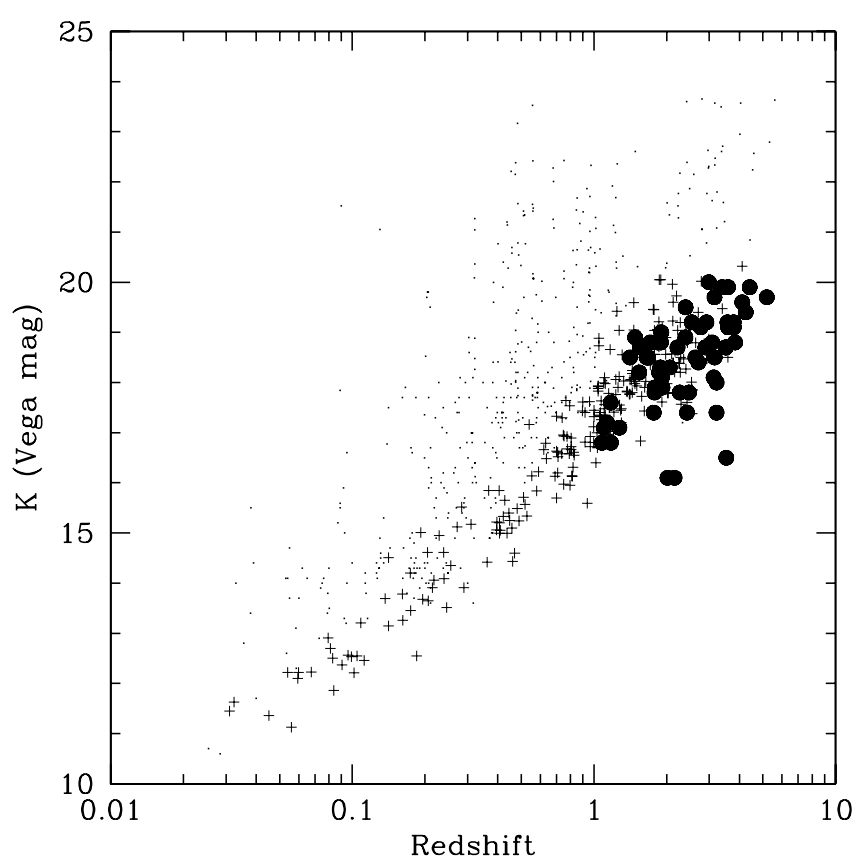

FIG. 1.- Hubble $K$-z diagram of radio-loud (pluses and filled circles) and radio-quiet galaxies ( points). The radio-loud sample is from De Breuck et al. (2002). The radio quiet samples are from the Hawaii survey (Songaila et al. 1994) and the Hubble Deep Field-North (Dickinson et al. 2003). The filled circles indicate the $69 z>1$ radio galaxies that comprise our Spitzer sample. The three HzRGs falling below the obvious correlation are MRC 0156-252 $(z=2.016)$, PKS $1138-262(z=2.156)$, and TX J1908+7220 $(z=3.530)$. MRC $0156-252$ is known to have strong $\mathrm{H} \alpha$ in the $K$ band (Eales \& Rawlings 1996), and PKS $1138-262$ has also recently been confirmed to have strong $\mathrm{H} \alpha$ in the $K$ band (Nesvadba et al. 2006). TX J1908+7220 has a strong unresolved AGN component (see notes in individual sources) and seems closer to being an unobscured quasar than a radio galaxy.

HzRGs with the most massive systems, however, is the tight correlation of the observed near-infrared Hubble, or $K-z$, diagram for powerful radio sources (Fig. 1; Lilly \& Longair 1984; Best et al. 1998; Eales et al. 1997; van Breugel et al. 1998; Jarvis et al. 2001; De Breuck et al. 2002; Willott et al. 2003; RoccaVolmerange et al. 2004; Brookes et al. 2006): HzRGs form a narrow redshift sequence that traces the envelope of radio-quiet galaxies and is well modeled by the evolution of a stellar population formed at high redshift from an accumulation of up to $10^{12} M_{\odot}$ of pregalactic baryonic material. The large-scale, doublelobed radio morphologies and enormous radio luminosities suggested early on that HzRGs must have spinning supermassive black holes powering relativistic jets in their centers (Rees 1978; Blandford \& Payne 1982). With the more recent discovery that the stellar bulge and central black hole masses of galaxies are closely correlated (e.g., Tremaine et al. 2002), it is no longer a surprise that the parent galaxies of the most powerful radio sources occupy the upper end of the galaxy mass function.

Despite two decades of study since the initial discovery of the HzRG $K-z$ relation (Lilly \& Longair 1984), the nature and tightness of the relation remains mysterious. The scatter in the relation is surprisingly low out to $z \sim 1$, and only increases modestly at higher redshifts as the observed $K$-band samples rest-frame optical emission (e.g., Fig. 1; De Breuck et al. 2002). Interpretations have generally relied on these observed near-IR observations probing the stellar populations of the distant radio galaxies (e.g., Rocca-Volmerange et al. 2004). However, detailed interpretations have been complicated by (1) uncertain contributions of AGNrelated light, which are most important in the rest-frame UV (e.g., Vernet et al. 2001), (2) evolution of the host galaxy stel-

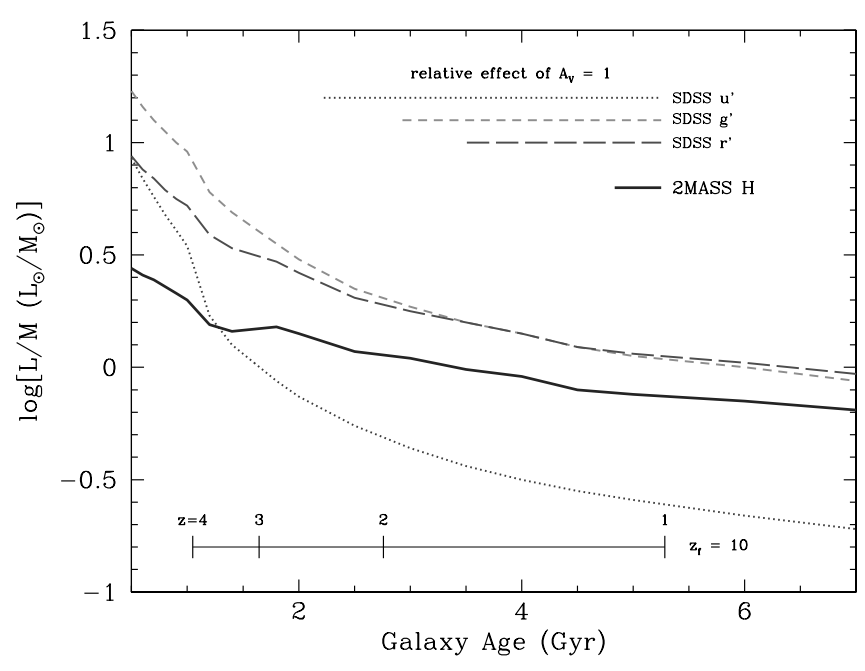

FIG. 2.-Light-to-mass ratios (in solar units) as a function of age for the adopted PÉGASE.2 elliptical galaxy model for different rest-frame optical (SDSS $\left.u^{\prime}, g^{\prime}, r^{\prime}\right)$ and near-IR (2MASS $H$ ) passbands. The light-to-mass ratio for the $H$-band filter, near the peak of stellar emission, varies much less as a function of age than the shorter wavelength filters. In addition, the optical passbands are more susceptible to (1) dust absorption - the relative effects of 1 mag of visual extinction in each of the passbands is illustrated by the horizontal lines; in units of $A_{\lambda}$, $H$ band is more than 4 times less sensitive to dust extinction than $r^{\prime}$, (2) uncertainty in the age of the stellar population, and (3) the addition of light from a second, younger population. For a formation redshift of $z_{\text {form }}=10$, the corresponding ages are marked near the bottom of the figure for different redshifts. For $z_{\text {form }}=14$ or 7 , the tracks shift 0.2 Gyr to the left or 0.3 Gyr to the right, respectively. As the $H$-band track is fairly flat, this uncertain formation redshift has little effect on our derived light-to-mass ratio. [See the electronic edition of the Supplement for a color version of this figure.]

lar population(s), and (3) band-shifting, meaning that observed $2.2 \mu \mathrm{m}$ samples very different emitting wavelengths for different redshift sources, reaching into the rest-frame ultraviolet for the most distant HzRGs.

In this paper, we present observations of a large sample of HzRGs obtained with the Spitzer Space Telescope (Werner et al. 2004). By observing the same rest-frame near- to mid-IR spectral range for sources over a large redshift range $(1<z<5.2)$, we remove many of the complications that plagued previous studies. In particular, the $1.6 \mu \mathrm{m}$ peak of the stellar emission provides a reasonably robust measure of the stellar mass for stellar populations with ages $\gtrsim 1$ Gyr (Sawicki 2002). While UV and optical emission has strong contributions from the youngest and hottest stars in a galaxy, near-IR emission primarily derives from the low-mass stars that dominate the stellar mass of a galaxy. Thus, while rest-frame UV and optical studies of galaxies are well-suited to probe galaxy star-formation rates, rest-frame near-IR studies are well-suited to probe galaxy stellar masses. We discuss this approach and other recent results that might affect it (e.g., Maraston 2005) in detail in $\S 6$.

Figure 2 illustrates the advantage of rest-frame near-IR derivations of stellar masses. First, the impact of dust extinction falls sharply with wavelength, making quantities derived from restframe near-IR observations more than an order of magnitude less susceptible to uncertain dust extinction corrections relative to optical observations. Second, since the main-sequence lifetimes of low-mass stars exceed the Hubble time, galaxy masses derived from rest-frame near-IR observations are relatively insensitive to the age of stellar populations or the star formation history. In particular, secondary bursts of star formation will affect UV and optical magnitudes significantly more than near-IR magnitudes.

We here use mid-IR observations of a large sample of radio galaxies to probe their host galaxy stellar masses. By observing 
consistently on both sides of the $1.6 \mu \mathrm{m}$ peak of the stellar emission, we avoid complicated $k$-correction effects and derive a reasonably robust measure of the stellar mass for stellar populations with ages $\gtrsim 1 \mathrm{Gyr}$. Observations at longer wavelengths allow us to determine the contribution of warm, AGN-heated dust emission to the rest-frame near-IR emission. Our paper is organized as follows. Section 2 describes the Spitzer HzRG sample. Section 3 presents the Spitzer mid- to far-IR data and their reduction. Section 4 describes our $\chi^{2}$ fitting of the spectral energy distributions (SEDs), and $\S 5$ presents the results of this fitting. Section 6 describes how the derived rest-frame $H$-band luminosities are converted into stellar masses. Section 7 discusses and concludes this analysis. We present notes on individual sources as an Appendix. This paper presents our entire Spitzer data set and analyzes the bulk properties of the sample. Spitzer observations of individual sources from this program have been the subject of detailed studies by Villar-Martín et al. (2006) of MRC 2104242 at $z=2.49$, by Stern et al. (2006) of LBDS 53W091 at $z=$ 1.55 , by Broderick et al. (2007) of PKS at $z=2.156$, and by C. De Breuck et al. (2007, in preparation) of 4C 23.56 at $z=2.48$. Throughout we assume a concordance model ${ }^{12}$ of universe expansion, $\Omega_{M}=1-\Omega_{\Lambda}=0.3, \Omega_{0}=1$, and $H_{0}=70 \mathrm{~km} \mathrm{~s}^{-1} \mathrm{Mpc}^{-1}$. Inferred luminosities presented in this paper are of the form $\nu L_{\nu} / L_{\odot}$, where $L_{\odot}=3.9 \times 10^{23} \mathrm{~W}$.

\section{THE SPITZER HIGH-REDSHIFT RADIO GALAXY SAMPLE}

Our HzRG sample is drawn from radio galaxy surveys executed during the last 45 years (starting with the 3CR; Bennett 1962). We have searched both flux-limited surveys such as the 3C (Spinrad et al. 1985), 6CE (Eales et al. 1997), 7C (Lacy et al. 1999; Willott et al. 2001), MG (Bennett et al. 1986; Lawrence et al. 1986), and MRC (McCarthy et al. 1996), as well as surveys filtered by their ultrasteep radio spectra (USS; e.g., Chambers et al. 1996; Röttgering et al. 1997; De Breuck et al. 2001). The former provide a sample unbiased in their radio properties but lack the high-redshift $(z>2)$ sources mainly found in the USS samples. Because the USS samples also probe fainter radio flux densities, including such sources allows us to break the strong luminosity-redshift degeneracy of flux density-limited surveys.

For the purposes of this work we define a HzRG as a radio galaxy above a redshift of one with a rest-frame $3 \mathrm{GHz}$ luminosity greater than $10^{26} \mathrm{WHz}^{-1}$. This choice is to ensure that we include only those objects with very powerful obscured AGNs. This value is similar to that used by other authors to separate AGNs based on their radio luminosities. The classical luminosity that separates Fanaroff \& Riley (1974) type 1 and type 2 sources (which are morphologically distinct), converted from $178 \mathrm{MHz}$ to $3 \mathrm{GHz}$, is $10^{25.4}\left(10^{24.8}\right) \mathrm{W} \mathrm{Hz}^{-1}$ assuming a steep (ultrasteep) radio spectral index of $\alpha=-0.8(-1.3)$. A more recent radioloud/radio-quiet division is $L_{3 \mathrm{GHz}}=10^{26.3}\left(10^{26.4}\right) \mathrm{W} \mathrm{Hz}^{-1}$ (Miller et al. 1990) and an upper limit to radio-quiet QSOs of $L_{3 \mathrm{GHz}}=10^{25.2}\left(10^{25.1}\right) \mathrm{W} \mathrm{Hz}^{-1}$ was found by Gregg et al. (1996) both converted to $3 \mathrm{GHz}$ luminosity assuming the same spectral index values.

To examine how Spitzer-derived quantities depend on redshift and radio luminosity, we selected a representative subset of

\footnotetext{
12 These common values of the "concordance" cosmology underestimate luminosities (and hence stellar masses) by $\sim 2 \%-3 \%$ at $z=3-4$ when compared to the latest Wilkinson Microwave Anisotropy Probe measurements, $\Omega_{M}=1-$ $\Omega_{\Lambda}=0.25, \Omega_{0}=1$, and $H_{0}=73 \mathrm{~km} \mathrm{~s}^{-1} \mathrm{Mpc}^{-1}$ (Spergel et al. 2007). Lower redshift HzRGs show an even smaller systematic adjustment, implying that the assumed cosmological parameters are inconsequential to our analysis.
}

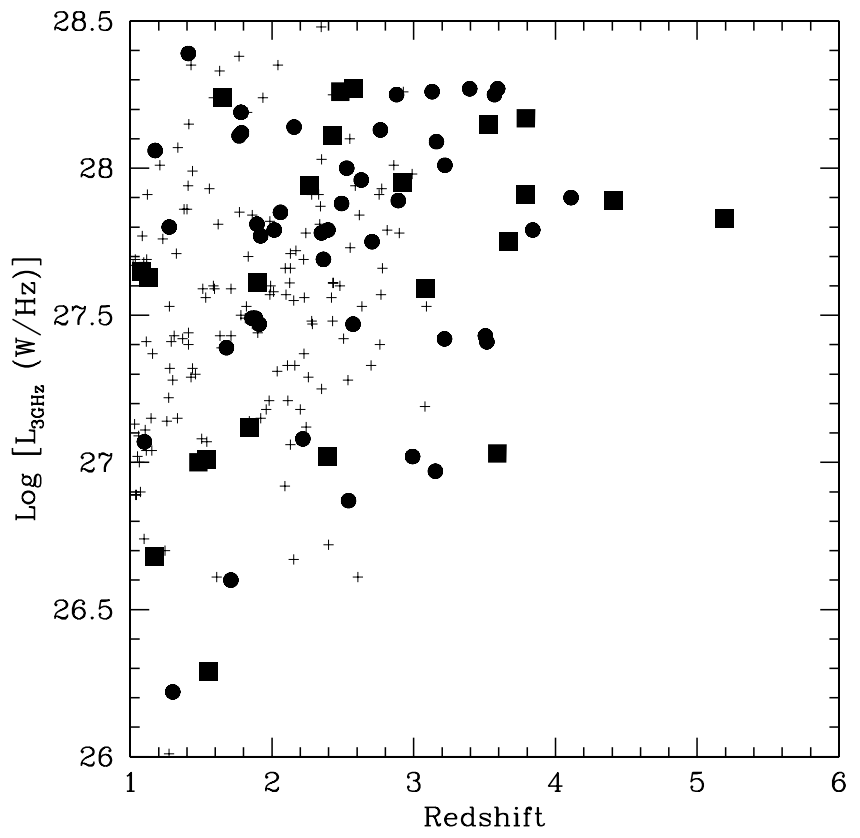

FIG. 3.- Radio luminosity at rest-frame $3 \mathrm{GHz}$ plotted against redshift for $263 z>1$ radio galaxies from the literature (pluses). Filled symbols indicate sources in our Spitzer program, carefully chosen to uniformly cover 1.5-2 orders of magnitude in radio power at each redshift $1<z<4$. The entire Spitzer sample was observed with IRAC. Radio galaxies at $z>2$ were imaged at $16 \mu \mathrm{m}$ with IRS. Radio galaxies in "low" Galactic infrared background (see $\S 3$ ) are indicated as solid squares and were observed with MIPS.

69 targets from the parent sample of 263 known $z>1$ HzRGs (circa 2003; Fig. 3). Table 1 lists the selected subset, which spans the full range of redshifts from $z=1$ to the redshift of the most distant known radio galaxy, TN J0924-2201 at $z=5.19$ (van Breugel et al. 1999). Most sources were observed as part of Spitzer Cycle 1 (Program ID number 3329); a few observations derive from guaranteed time observations by Spitzer instrument teams. We required at least 15 sources in each $\Delta z=1$ bin, covering a range of rest-frame $3 \mathrm{GHz}$ radio luminosity, $L_{3 \mathrm{GHz}}$. To obtain a uniform set of radio luminosities of a sample of radio galaxies covering the entire sky, we obtained radio flux densities from the largest all-sky radio surveys, i.e., the 1.4 GHz NRAO VLA Sky Survey (NVSS: Condon et al. 1998), the 325/352 MHz Westerbork Northern Sky Survey (WENSS: Rengelink et al. 1997), and the $365 \mathrm{MHz}$ Texas Survey (Douglas et al. 1996). These surveys all have $\sim 1^{\prime}$ spatial resolution, which has the advantage that the entire sample appear essentially as point sources, thereby minimizing issues of missing flux from overresolved emission. We chose $3 \mathrm{GHz}$ as the fiducial rest frequency as it is the midpoint between the survey frequencies at the median redshift of our sample. This choice ensures that almost all radio luminosities are interpolated, rather than extrapolated as is often required when deriving lower rest frequency luminosities (e.g., 151 or $178 \mathrm{MHz}$ ). Blundell et al. (1998) argue, however, that low-frequency luminosities are a better measure of the total radio luminosity, as the emission at low frequency is dominated by the steep-spectrum, isotropic radio lobes, while high-frequency emission may be contaminated by Doppler-boosted emission from flat-spectrum radio cores. Until the completion of all-sky $<100 \mathrm{MHz}$ surveys, such low-frequency rest-frame luminosities cannot be uniformly derived for our sample. However, most HzRGs in our sample have weak radio cores, which although flatter than the ultrasteep-spectrum radio lobes, still have steep radio spectra (e.g., Athreya et al. 1997) and thus are not expected to significantly boost the rest-frame $3 \mathrm{GHz}$ luminosity. 
TABLE 1

Spitzer HzRG Sample and Exposure Times per Instrument

\begin{tabular}{|c|c|c|c|c|c|c|c|c|}
\hline $\mathrm{HzRG}$ & R.A. (J2000.0) & Decl. (J2000.0) & Redshift & $\begin{array}{l}\log \left(L_{3 \mathrm{GHz}}\right) \\
\left(\mathrm{W} \mathrm{Hz}^{-1}\right)\end{array}$ & $K$ Band $^{\mathrm{a}}$ (Vega) & $\begin{array}{l}\text { IRAC Exp. Time } \\
\text { (s) }\end{array}$ & $\begin{array}{l}\text { IRS Exp. Time } \\
\text { (s) }\end{array}$ & $\begin{array}{l}\text { MIPS Exp. Time } \\
\text { (s) }\end{array}$ \\
\hline $6 \mathrm{C} 0032+412 \ldots \ldots \ldots \ldots$ & 003453.1 & 413131.50 & 3.670 & 27.75 & 19.10 & 120 & 122 & $134,420,881$ \\
\hline $6 C^{*} 0058+495 \ldots \ldots \ldots . .$. & 010118.9 & 495012.29 & 1.173 & 26.68 & 17.60 & 120 & $\ldots$ & $134,420,881$ \\
\hline MRC $0114-211 \ldots \ldots .$. & 011651.4 & -205206.71 & 1.410 & 28.39 & 18.50 & 120 & $\ldots$ & $\ldots$ \\
\hline TN J0121+1320_......... & 012142.7 & 132058.00 & 3.516 & 27.41 & 18.70 & 120 & 122 & $\ldots$ \\
\hline MRC $0152-209 \ldots \ldots .$. & 015455.8 & -204026.30 & 1.920 & 27.77 & 17.90 & 120 & & $\ldots$ \\
\hline MRC $0156-252 \ldots \ldots .$. & 015833.6 & -245931.10 & 2.016 & 27.79 & 16.10 & 120 & 122 & $\ldots$ \\
\hline TN J0205+2242......... & $0205 \quad 10.7$ & 224250.40 & 3.506 & 27.43 & 18.70 & 120 & 122 & $\ldots$ \\
\hline MRC $0211-256 \ldots \ldots .$. & 021330.5 & -252521.00 & 1.300 & 26.22 & $\ldots$ & 120 & 122 & $\ldots$ \\
\hline 3C 65 & 022343.2 & 400052.40 & 1.176 & 28.06 & 16.80 & 120 & $\ldots$ & $\ldots$ \\
\hline MRC $0251-273 \ldots \ldots .$. & 025316.7 & -270913.03 & 3.160 & 28.09 & 18.50 & 120 & 122 & $\ldots$ \\
\hline MRC $0316-257 \ldots \ldots .$. & 031812.0 & -253511.00 & 3.130 & 28.26 & 18.10 & 120 & 122 & $\ldots$ \\
\hline PKS $0529-549 \ldots \ldots \ldots . .$. & 053025.2 & -545422.00 & 2.575 & 28.27 & $\ldots$ & 120 & 122 & $134,420,881$ \\
\hline WN J0617+5012 ....... & 061739.4 & 501255.40 & 3.153 & 26.97 & 19.70 & 120 & 122 & $\ldots$ \\
\hline 4C $41.17 \ldots \ldots \ldots \ldots \ldots \ldots$ & 065052.1 & 413031.00 & 3.792 & 28.17 & 19.10 & 5000 & 122 & $267,671,2643$ \\
\hline WN J0747+3654 ....... & 074729.4 & 365438.09 & 2.992 & 27.02 & 20.00 & 120 & 122 & $\ldots$ \\
\hline 6 CE $0820+3642 \ldots \ldots .$. & 082348.1 & 363246.42 & 1.860 & 27.49 & 18.20 & 120 & $\ldots$ & $\ldots$ \\
\hline USS $0828+193 \ldots \ldots \ldots$ & 083053.4 & 191316.00 & 2.572 & 27.47 & 18.20 & 120 & 122 & $\ldots$ \\
\hline 5C 7.269 & 082838.8 & 252827.10 & 2.218 & 27.08 & 18.90 & 120 & 122 & $\ldots$ \\
\hline 6 CE $0901+3551 \ldots \ldots .$. & 090432.4 & 353903.23 & 1.910 & 27.47 & 18.10 & 120 & $\ldots$ & $\ldots$ \\
\hline 6CE $0905+3955 \ldots \ldots .$. & 090816.9 & 394326.00 & 1.883 & 27.49 & 18.30 & 120 & $\ldots$ & $\ldots$ \\
\hline B2 $0902+34 \ldots \ldots \ldots \ldots$ & 090530.1 & 340756.89 & 3.395 & 28.27 & 19.90 & 1200 & 122 & $2557,2696,3556$ \\
\hline TN J0924-2201 ......... & 092419.9 & -220141.00 & 5.195 & 27.83 & 19.70 & 120 & 122 & $134,420,881$ \\
\hline $6 \mathrm{C} 0930+389 \ldots \ldots \ldots \ldots$ & 093306.9 & 384150.14 & 2.395 & 27.79 & 19.50 & 120 & 122 & $\ldots$ \\
\hline USS $0943-242 \ldots \ldots \ldots . .$. & 094532.7 & -242849.65 & 2.923 & 27.95 & 19.20 & 120 & 122 & $134,420,881$ \\
\hline $6 \mathrm{C} 1232+39 \ldots \ldots \ldots \ldots \ldots$ & 123504.8 & 392538.91 & 3.220 & 28.01 & 18.00 & 120 & 122 & $\ldots$ \\
\hline USS $1243+036 \ldots \ldots \ldots . .$. & 124538.4 & 32320.70 & 3.570 & 28.25 & 19.20 & 120 & 122 & $\ldots$ \\
\hline TN J1338-1942 …..... & 133826.0 & -194231.00 & 4.110 & 27.90 & 19.60 & 5000 & 122 & $\ldots$ \\
\hline 4C $24.28 \ldots \ldots \ldots \ldots \ldots . .$. & 134814.8 & 241552.00 & 2.879 & 28.25 & $\ldots$ & 120 & 122 & $\ldots$ \\
\hline 3C 294.0 & 140644.0 & 341125.00 & 1.786 & 28.12 & 17.90 & 120 & $\ldots$ & $\ldots$ \\
\hline USS $1410-001 \ldots \ldots \ldots . .$. & 141315.1 & 02259.70 & 2.363 & 27.69 & $\ldots$ & 120 & 122 & $\ldots$ \\
\hline $8 \mathrm{C} 1435+635 \ldots \ldots \ldots \ldots$ & 143637.1 & 631914.00 & 4.250 & 28.55 & 19.40 & 120 & 122 & $134,420,881$ \\
\hline USS $1558-003 \ldots \ldots \ldots . .$. & 160117.3 & 02848.00 & 2.527 & 28.00 & $\ldots$ & 120 & 122 & $\ldots$ \\
\hline USS $1707+105 \ldots \ldots \ldots$ & 171006.5 & 103106.00 & 2.349 & 27.78 & $\ldots$ & 120 & 122 & $\ldots$ \\
\hline LBDS 53w002 .............. & 171414.7 & 501529.70 & 2.393 & 27.02 & 18.90 & 3300 & 122 & $134,420,881$ \\
\hline LBDS 53w091 .............. & 172232.7 & 500601.94 & 1.552 & 26.29 & 18.70 & 900 & $\ldots$ & $633,1311,2643$ \\
\hline 3C 356.0 & 172419.0 & 505740.30 & 1.079 & 27.65 & 16.80 & 120 & $\ldots$ & $134,420,881$ \\
\hline 7C $1751+6809 \ldots \ldots \ldots$ & 175049.9 & 680825.93 & 1.540 & 27.01 & 18.20 & 120 & $\ldots$ & $134,420,881$ \\
\hline 7C $1756+6520 \ldots \ldots \ldots . .$. & 175705.4 & 651953.11 & 1.480 & 27.00 & 18.90 & 120 & $\ldots$ & $134,420,881$ \\
\hline 3C $368.0 \ldots \ldots \ldots \ldots \ldots$ & 180506.3 & 110133.00 & 1.132 & 27.63 & 17.20 & 120 & $\ldots$ & $134,420,881$ \\
\hline 7C $1805+6332 \ldots \ldots \ldots . .$. & 180556.9 & $6333 \quad 13.14$ & 1.840 & 27.12 & 18.80 & 120 & $\ldots$ & $134,420,881$ \\
\hline 4C 40.36 & 181055.7 & 404524.01 & 2.265 & 27.94 & 17.80 & 120 & 122 & $134,420,881$ \\
\hline TX J1908+7220_......... & 190823.7 & $72 \quad 2011.82$ & 3.530 & 28.15 & 16.50 & 120 & 122 & $134,420,881$ \\
\hline WN J1911+6342 ....... & 191149.6 & 634209.60 & 3.590 & 27.03 & 19.90 & 120 & 122 & $134,420,881$ \\
\hline TN J2007-1316 ......... & 200753.3 & -131643.62 & 3.840 & 27.79 & 18.80 & 120 & 122 & $\ldots$ \\
\hline MRC $2025-218 \ldots \ldots \ldots$ & 202759.5 & -214056.90 & 2.630 & 27.96 & 18.50 & 120 & 122 & $\ldots$ \\
\hline MRC $2048-272 \ldots \ldots \ldots$ & 205103.6 & -270302.53 & 2.060 & 27.85 & 18.30 & 120 & 122 & $\ldots$ \\
\hline MRC $2104-242 \ldots \ldots . .$. & 210658.1 & -240511.00 & 2.491 & 27.88 & $\ldots$ & 120 & 122 & $\ldots$ \\
\hline 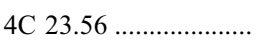 & 210714.8 & 233145.00 & 2.483 & 28.26 & 19.73 & 120 & 122 & $134,420,881$ \\
\hline MG 2144+1928 .......... & 214407.5 & 192914.60 & 3.592 & 28.27 & 19.10 & 120 & 122 & $\ldots$ \\
\hline
\end{tabular}


TABLE 1 - Continued

\begin{tabular}{|c|c|c|c|c|c|c|c|c|}
\hline HzRG & R.A. (J2000.0) & Decl. (J2000.0) & Redshift & $\begin{array}{l}\log \left(L_{3 \mathrm{GHz}}\right) \\
\left(\mathrm{W} \mathrm{Hz}^{-1}\right)\end{array}$ & $K$ Band $^{\mathrm{a}}$ (Vega) & $\begin{array}{l}\text { IRAC Exp. Time } \\
\text { (s) }\end{array}$ & $\begin{array}{l}\text { IRS Exp. Time } \\
\text { (s) }\end{array}$ & $\begin{array}{l}\text { MIPS Exp. Time } \\
\text { (s) }\end{array}$ \\
\hline USS $2202+128 \ldots \ldots \ldots . .$. & 220514.1 & 130533.50 & 2.706 & 27.75 & 18.40 & 120 & 122 & . \\
\hline B3 J2330+3927 .......... & 233024.9 & 392712.02 & 3.086 & 27.59 & 18.80 & 120 & 122 & $134,420,881$ \\
\hline 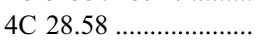 & 235159.2 & 291028.99 & 2.891 & 27.89 & 18.70 & 120 & 122 & $\ldots$ \\
\hline 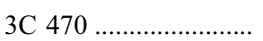 & 235835.3 & 440438.87 & 1.653 & 28.24 & 18.50 & 120 & $\ldots$ & $134,420,881$ \\
\hline
\end{tabular}

NoтE.- -Units of right ascension are hours, minutes, and seconds, and units of declination are degrees, arcminutes, and arcseconds.

${ }^{a} K$-band magnitude within projected 64 kpc radius.

We have preferentially selected sources with the most supporting data. Forty-seven of the $69 \mathrm{HzRGs}$ in our sample have HST data, representing over $1.5 \mathrm{Ms}$ of HST time. We also preferentially included sources previously selected for guaranteed time observations (GTOs) by the Spitzer instrument teams, thereby minimizing our Spitzer observatory request while maintaining a uniformly selected sample. Finally, we preferentially selected sources with millimeter and submillimeter observations in order to have information on the SEDs longward of the Spitzer coverage. Note that the sample does not suffer a bias toward cold dust properties as we select only on the basis of (sub-) millimeter observations, not detections.

\section{INFRARED OBSERVATIONS AND REDUCTIONS}

The Spitzer observations consist of imaging photometry in eight bands, using the entire instrument complement of the observatory. Data from the Infrared Array Camera (IRAC; Fazio et al. 2004) comes from all four bands $(3.6,4.5,5.8$, and $8 \mu \mathrm{m}$ - synonymously referred to as channels $1,2,3$, and 4$)$. The entire sample of $69 \mathrm{HzRGs}$ was observed with IRAC. ${ }^{13}$ Data from the Infrared Spectrograph (IRS; Houck et al. 2004) obtained in "peak-up imaging" mode (cf., Teplitz et al. 2005) provides flux densities at $16 \mu \mathrm{m}$ for the 46 objects in our sample at $z>2$. For the lower redshift portion of our sample, IRAC $8 \mu \mathrm{m}$ images adequately cover the SED longward of the $1.6 \mu \mathrm{m}$ stellar bump. Finally, the Multiband Imaging Photometer for Spitzer (MIPS; Rieke et al. 2004) was used to image a subset of our sample using all three available bands $(24,70$, and $160 \mu \mathrm{m})$. Due to the uncertain ability of MIPS to image against the Galactic infrared background at the time of our GO Cycle 1 submission, only the 26 HzRGs from our sample lying in "low" background regions $\left(S_{24 \mu \mathrm{m}}<\right.$ $20 \mathrm{MJy} \mathrm{sr}^{-1}$ ) were observed with MIPS. Table 1 presents the 69 HzRGs comprising our sample, along with their coordinates, redshifts, rest-frame $3 \mathrm{GHz}$ radio luminosities, $64 \mathrm{kpc} K$-band magnitudes (Vega), and which Spitzer instruments they have been observed with.

The 141 unique target/instrument observations comprising our survey were released from the Spitzer archive between 2004 October and 2006 June. Fourteen observations were part of the initial GTO programs (e.g., Stern et al. 2006). In general, our data reduction begins with the basic calibrated data (BCD) output of the Spitzer Science Center pipeline, using the most current version number at the time the data were initially released (e.g., S9 to S13). Improvements over the generations of pipelines implemented here were minor and did not warrant reprocessing data. The most useful augmentation was the addition of a global world coordinate system to the IRS peak-up images in S11. Note that

\footnotetext{
13 Although 3C 65 was observed in only two bands due to an astrometric error in NED, which we have since had corrected.
}

the units of Spitzer BCD images are in surface brightness units of $\mathrm{MJy} \mathrm{sr}^{-1}$, where $1 \mathrm{MJy} \mathrm{sr}^{-1}$ corresponds to $23.5 \mu \mathrm{Jy} \operatorname{arcsec}^{-2}$.

\subsection{IRAC OBSERVATIONS}

Most of the IRAC observations consisted of four dithered $30 \mathrm{~s}$ exposures in each of the four IRAC channels. For each channel, the BCDs were mosaicked using the MOPEX package (Makovoz $\&$ Khan 2005) from the Spitzer Science Center and resampled by a factor of 2 to give a pixel scale of $0.61^{\prime \prime}$. The MOPEX outlier (e.g., cosmic ray, bad pixel) rejection was optimized for the regions of deepest coverage in the center of the maps, corresponding to the location of the targeted HzRGs. The eight GTO IRAC observations were all deeper than our standardized program and were reduced in a similar manner with MOPEX. Source extraction was performed with SExtractor (Bertin \& Arnouts 1996) in dual image mode, using the $3.6 \mu \mathrm{m}$ channel for source detection. All IRAC BCD images have astrometry derived from 2 MASS survey and are good to $<0.5^{\prime \prime}$, so we can be confident of the radio galaxy identifications.

We generally used the a $7^{\prime \prime}$ diameter aperture for photometry, although in a few cases where the source was faint and/or had a close companion, we extracted photometry using a $3.5^{\prime \prime}$ diameter aperture. The aperture corrections, applied after the conversion from surface brightness to flux density, are listed in Table 2 and are taken from the Spitzer First Look Survey (Lacy et al. 2005). The resulting flux densities are listed in Table 3 and include the total uncertainty in the IRAC photometry; i.e., the statistical uncertainties are added in quadrature to the systematic uncertainty of $\sim 10 \%$. The systematic uncertainty is the total uncertainty from several different effects: the uncertainty in the IRAC flux density calibration, a flat-fielding color-dependent uncertainty, and variation of the PSF across the field of view. Nondetections have $3 \sigma$ upper limits listed. The statistical uncertainties in the mosaicked images were calculated from the pixel-to-pixel rms from single BCDs (with the native pixel scale), converted to $\mu \mathrm{Jy}$, multiplied by the square root of the area of the aperture in square pixels, divided by the square root of the number of frames, and multiplied by the appropriate aperture correction. In all cases, the radio galaxies are well detected in the 3.6 and $4.5 \mu$ m channels. ${ }^{14}$ In most cases, the radio galaxies are also detected in the 5.8 and $8 \mu \mathrm{m}$ channels. In no cases were the HzRGs resolved in channel 1 , the highest resolution of all the Spitzer imaging bands with a PSF FWHM of $1.44^{\prime \prime}$.

\subsection{IRS OBSERVATIONS}

The IRS observations consisted of just two nodded exposures of the $16 \mu \mathrm{m}$ peak-up imager, each of $61 \mathrm{~s}$ duration. The observations

$143 \mathrm{C} 65$ is not observed in the $4.5 \mu$ m channel. $6 \mathrm{C} 0140+326$ is probably detected at 3.6 and $4.5 \mu \mathrm{m}$ but is blended by a foreground galaxy. Likewise 3C 294 is detected in all four channels, but the proximity of a bright star means only upper limits can be determined for channels at 3.6 and $4.5 \mu \mathrm{m}$. 
TABLE 2

Spitzer Photometric Parameters

\begin{tabular}{|c|c|c|c|c|c|c|}
\hline Filter & $\begin{array}{l}\text { Wavelength } \\
\qquad(\mu \mathrm{m})\end{array}$ & $\begin{array}{c}\text { Pixel Scale } \\
\left(\operatorname{arcsec} \text { pixel }^{-1}\right)\end{array}$ & Conv. to $\mu \mathrm{Jy}$ & $\begin{array}{l}\text { Aperture }^{a} \\
(\operatorname{arcsec})\end{array}$ & $\begin{array}{l}\text { Annulus }^{\mathrm{b}} \\
(\operatorname{arcsec})\end{array}$ & $\begin{array}{l}\text { Aperture } \\
\text { Correction }\end{array}$ \\
\hline \multirow[t]{2}{*}{ 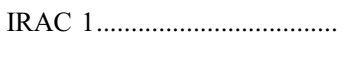 } & 3.6 & 0.61 & 23.5 & 3.5 & 9.76 & 1.48 \\
\hline & & & & 7.0 & 9.76 & 1.13 \\
\hline \multirow[t]{2}{*}{ 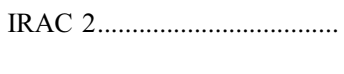 } & 4.5 & 0.61 & 23.5 & 3.5 & 9.76 & 1.60 \\
\hline & & & & 7.0 & 9.76 & 1.168 \\
\hline \multirow[t]{2}{*}{ 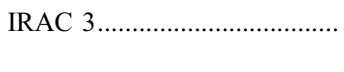 } & 5.8 & 0.61 & 23.5 & 3.5 & 9.76 & 2.76 \\
\hline & & & & 7.0 & 9.76 & 1.155 \\
\hline \multirow[t]{2}{*}{ IRAC $4 \ldots \ldots \ldots \ldots \ldots \ldots \ldots \ldots \ldots \ldots \ldots \ldots \ldots$} & 8.0 & 0.61 & 23.5 & 3.5 & 9.76 & 2.13 \\
\hline & & & & 7.0 & 9.76 & 1.316 \\
\hline 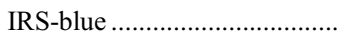 & 16 & 1.2 & $0.591^{\mathrm{c}}$ & 6 & $6-10$ & $\ldots$ \\
\hline MIPS 1 & 24 & 1.25 & 36.71 & 13 & $20-32$ & 1.167 \\
\hline 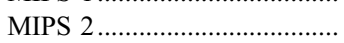 & 70 & 4.95 & 576 & 35 & $39-65$ & $1.211^{\mathrm{d}}$ \\
\hline MIPS 3 & 160 & 8 & 1500 & 50 & $75-125$ & 1.445 \\
\hline
\end{tabular}

\footnotetext{
a Diameter at which the photometry is measured.

b Inner and outer diameter of annulus used to determine the background sky level, except for the IRAC bands, where we provide the size of the background mesh used by SExtractor.

c Including aperture correction.

d For a power-law SED, $f_{\nu} \propto \nu^{-2}$
}

were executed in Spitzer Cycle 1, before IRS "peak-up imaging" was offered as a supported observing mode. However, all the data were reduced with the more recent S13.2.0 version of the archive pipeline, which provides astrometry to the $\mathrm{BCD}$ products accurate to $0.5^{\prime \prime}$ relative to the $2 \mathrm{MASS}$ reference frame. Subimages were made of just the blue $(16 \mu \mathrm{m})$ "peak-up" field of view in which the radio galaxy was centered. Difference images were then created by subtracting one frame from the other, thereby removing background emission and latent patterns in the array.

The dithered difference images were then combined with bad pixels and flaring pixels masked. Flux densities were extracted with the IRAF DAOPHOT task using the parameters listed in Table 2. Random uncertainties were determined from the rms in each BCD and converted to $\mu \mathrm{Jy}$. The total uncertainties in this table include the random and the 7.8\% (6\% zero point uncertainty and $5 \%$ color correction) systematic uncertainties. The systematic and random uncertainties are combined in quadrature and used for the SED fitting discussed in $\S 4$. These flux densities and uncertainties are listed in Table 3 . Nondetections are represented by $3 \sigma$ upper limits. All 46 of the Spitzer HzRG sample at $z \geq 2$ were observed with IRS, as was MRC $0211-256(z=1.300)$ due to an error in the submitted AORs. ${ }^{15}$ We have robust $16 \mu \mathrm{m}$ detections of the radio galaxy in 35 of the 47 observations. ${ }^{16}$

\subsection{MIPS OBSERVATIONS}

The MIPS observations were restricted to the $26 \mathrm{HzRG}$ with "low" mid-IR background $\left(S_{24 \mu \mathrm{m}}<20 \mathrm{MJy} \mathrm{sr}^{-1}\right)$ and consisted of one cycle of $30 \mathrm{~s}$ exposures at $24 \mu \mathrm{m}$, three cycles of $10 \mathrm{~s} \mathrm{ex}-$ posures at $70 \mu \mathrm{m}$, and five cycles of $10 \mathrm{~s}$ exposures at $160 \mu \mathrm{m}$. The $24 \mu \mathrm{m}$ BCDs were median filtered to remove a regular stripe artifact pattern prior to mosaicking. Both the 24 and $70 \mu \mathrm{m}$ data were then mosaicked with MOPEX using standard inputs and with the pixels resampled by a factor of 2 . Twenty-two of the $24 \mu \mathrm{m}$ observations resulted in detections, but only four radio galaxies are detected at $70 \mu \mathrm{m}$. None of our HzRG sample are detected at $160 \mu \mathrm{m}$. As we shall see below, the upper limits at long

\footnotetext{
15 It was inadvertently confused with USS 0211-122 $(z=2.336)$.

16 Except for the nondetection of the highest redshift radio galaxy, TN J0924-2201 ( $z=5.195)$, the $16 \mu$ m nondetections are evenly distributed across $z \sim 2-4$.
}

wavelengths are still quite useful for constraining the mid-IR SED. Flux densities were extracted with the IRAF DAOPHOT task using the parameters listed in Table 2 . For the $70 \mu \mathrm{m}$ band we use an aperture correction based on a steep spectrum $\left(F_{\lambda} \propto \lambda^{0}\right)$. Again, Table 3 provides flux densities and upper limits $(3 \sigma)$ for all three MIPS cameras.

The random uncertainties (and $3 \sigma$ upper limits in Table 3 ) are derived from the BCDs and converted to $\mu \mathrm{Jy}$. The color correction can be quite significant for these broad MIPS bands that were calibrated using Galactic stars, e.g., typically for a Raleigh-Jeans spectrum, $F_{\lambda} \propto \lambda^{-4}$. For the 70 and $160 \mu \mathrm{m}$ bands we include a $5 \%$ systematic uncertainty to account for the uncertain color correction. For $24 \mu \mathrm{m}$ we typically find that the SED is very nonstellar. As a first approximation, we apply the color correction by multiplying by 0.961 (from the MIPS data handbook ${ }^{17}$ ) for $\lambda F_{\lambda}=$ constant, which approximates the observed SEDs of our HzRGs. We further include a $2 \%$ systematic uncertainty for the remaining unknown color correction. The final systematic uncertainties are $4.5 \%, 8.6 \%$, and $13 \%$ for 24,70 , and $160 \mu \mathrm{m}$, respectively, including flux-calibration uncertainties from the Spitzer Observers Manual and color correction uncertainties described above. The systematic uncertainties are combined in quadrature with the statistical uncertainties and are presented in Table 3.

\subsection{Mid-IR Colors of HzRGS}

Recent work has shown that IRAC mid-IR colors reliably isolate spectroscopically confirmed, luminous, unobscured AGNs (e.g., Lacy et al. 2004; Stern et al. 2005). However, since the surface density of sources with mid-IR colors similar to unobscured AGNs is much higher than that of optically selected (type 1) quasars, it has been inferred that obscured, type 2 quasars are likewise identifiable from their mid-IR colors. Due to their optical faintness, type 2 quasars are not as well studied as their bright, type 1 cousins. However, they are a natural consequence of AGN unification schemes (e.g., Antonucci 1993), and models of the hard X-ray background imply that they outnumber type 1 quasars by factors of several (e.g., Treister et al. 2004).

Our Spitzer HzRG sample represents the largest sample of confirmed type 2 quasars with IRAC photometry obtained to date.

${ }^{17} \mathrm{See}$ http://ssc.spitzer.caltech.edu/mips/dh/mipsdatahandbook3.2.pdf. 
TABLE 3

Spitzer Photometry for HzRGs $(\mu \mathrm{Jy})$

\begin{tabular}{|c|c|c|c|c|c|c|c|c|}
\hline HzRG & $f_{3.6 \mu \mathrm{m}}$ & $f_{4.5 \mu \mathrm{m}}$ & $f_{5.8 \mu \mathrm{m}}$ & $f_{8.0 \mu \mathrm{m}}$ & $f_{16 \mu \mathrm{m}}$ & $f_{24 \mu \mathrm{m}}$ & $f_{70 \mu \mathrm{m}}$ & $f_{160 \mu \mathrm{m}}$ \\
\hline $6 \mathrm{C} 0032+412 \ldots \ldots \ldots \ldots \ldots \ldots \ldots$ & $15.4 \pm 2.1$ & $33.8 \pm 3.7$ & $57.6 \pm 8.9$ & $98.3 \pm 10.0$ & $205.0 \pm 34.9$ & $457.0 \pm 59.7$ & $<3950$ & $<96000$ \\
\hline MR C0037-258 ................. & $221.0 \pm 22.0$ & $248.0 \pm 25.0$ & $286.0 \pm 29.0$ & $518.0 \pm 52.0$ & $\ldots$ & $\ldots$ & $\ldots$ & $\ldots$ \\
\hline $6 C^{*} 0058+495 \ldots \ldots \ldots \ldots \ldots \ldots$ & $82.0 \pm 8.3$ & $86.7 \pm 8.8$ & $93.2 \pm 9.5$ & $309.0 \pm 31.0$ & $\ldots$ & $1450.0 \pm 87.3$ & $18900 \pm 1962$ & $<94600$ \\
\hline MRC 0114-211 .................. & $87.3 \pm 8.9$ & $117.0 \pm 12.0$ & $157.0 \pm 16.0$ & $398.0 \pm 40.0$ & $\ldots$ & $\ldots$ & $\ldots$ & $\ldots$ \\
\hline TN J0121+1320................. & $10.5 \pm 1.8$ & $14.4 \pm 2.1$ & $<32.5$ & $<40.3$ & $<136.0$ & $\ldots$ & $\ldots$ & $\ldots$ \\
\hline $6 C^{*} 0132+330 \ldots \ldots \ldots \ldots \ldots \ldots . .$. & $31.6 \pm 3.6$ & $41.3 \pm 4.5$ & $51.9 \pm 12.4$ & $109.0 \pm 11.0$ & $\ldots$ & $\ldots$ & $\ldots$ & $\ldots$ \\
\hline 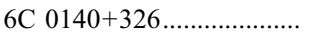 & $<623.0$ & $<450.0$ & $34.9 \pm 2.7$ & $36.3 \pm 3.4$ & $194.0 \pm 40.0$ & $2190.0 \pm 110.5$ & $<2580$ & $<20700$ \\
\hline MRC 0152-209 ................ & $108.0 \pm 11.0$ & $165.0 \pm 17.0$ & $215.0 \pm 22.0$ & $415.0 \pm 42.0$ & $\ldots$ & $\ldots$ & $\ldots$ & $\ldots$ \\
\hline MRC $0156-252 \ldots \ldots \ldots \ldots . . . .$. & $291.0 \pm 29.0$ & $405.0 \pm 41.0$ & $717.0 \pm 72.0$ & $1125.0 \pm 113.0$ & $1430.0 \pm 116.6$ & $\ldots$ & $\ldots$ & $\ldots$ \\
\hline TN J0205+2242 ................ & $7.5 \pm 1.7$ & $4.7 \pm 2.3$ & $<29.3$ & $<37.4$ & $<122.0$ & $\ldots$ & $\ldots$ & $\ldots$ \\
\hline MRC $0211-256 \ldots \ldots \ldots \ldots \ldots . . .$. & $166.0 \pm 17.0$ & $197.0 \pm 20.0$ & $222.0 \pm 22.0$ & $278.0 \pm 28.0$ & $330.0 \pm 42.6$ & $\ldots$ & $\ldots$ & $\ldots$ \\
\hline 3C $65 \ldots \ldots \ldots \ldots \ldots \ldots \ldots \ldots \ldots \ldots \ldots \ldots \ldots \ldots \ldots \ldots$ & $161.0 \pm 32.0$ & $\ldots$ & $320.0 \pm 66.0$ & $\ldots$ & $\ldots$ & $\ldots$ & $\ldots$ & $\ldots$ \\
\hline MRC $0251-273 \ldots \ldots \ldots \ldots \ldots . .$. & $11.5 \pm 1.6$ & $12.7 \pm 1.8$ & $<29.1$ & $<28.8$ & $97.2 \pm 34.2$ & $\ldots$ & $\ldots$ & $\ldots$ \\
\hline MRC $0316-257$................ & $19.3 \pm 2.1$ & $20.1 \pm 2.1$ & $19.5 \pm 2.4$ & $38.1 \pm 4.1$ & $<91.5$ & $\ldots$ & $\ldots$ & $\ldots$ \\
\hline MRC $0324-228 \ldots \ldots \ldots \ldots \ldots . . .$. & $39.4 \pm 4.2$ & $39.7 \pm 4.3$ & $61.1 \pm 8.6$ & $89.9 \pm 9.9$ & $\ldots$ & $\ldots$ & $\ldots$ & $\ldots$ \\
\hline 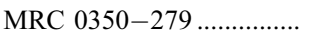 & $23.6 \pm 2.6$ & $40.6 \pm 4.2$ & $82.2 \pm 27.6$ & $79.3 \pm 26.5$ & $\ldots$ & $321.0 \pm 60.7$ & $<1040$ & $<88800$ \\
\hline MRC $0406-244 \ldots \ldots \ldots \ldots . . . . .$. & $40.4 \pm 4.3$ & $43.3 \pm 4.6$ & $<51.6$ & $63.5 \pm 14.5$ & $242.0 \pm 34.6$ & $1420.0 \pm 83.7$ & $24700 \pm 2306$ & $<47700$ \\
\hline 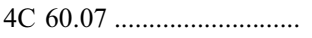 & $27.3 \pm 3.1$ & $33.6 \pm 3.7$ & $35.1 \pm 8.4$ & $37.2 \pm 9.5$ & $175.0 \pm 33.0$ & $1340.0 \pm 83.7$ & $<3750$ & $<64600$ \\
\hline 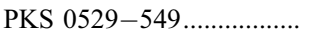 & $46.6 \pm 4.9$ & $52.9 \pm 5.5$ & $62.7 \pm 8.6$ & $72.2 \pm 9.1$ & $134.0 \pm 33.7$ & $942.0 \pm 71.0$ & $<4110$ & $<74100$ \\
\hline WN J0617+5012 …............. & $3.6 \pm 1.0$ & $5.5 \pm 1.2$ & $<48.3$ & $<54.5$ & $<93.4$ & $\ldots$ & $\ldots$ & $\ldots$ \\
\hline 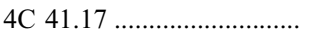 & $23.4 \pm 2.4$ & $27.5 \pm 2.8$ & $35.6 \pm 3.7$ & $36.5 \pm 3.5$ & $<99.3$ & $370.0 \pm 43.3$ & $<3210$ & $<26300$ \\
\hline WN J0747+3654 „.............. & $19.1 \pm 2.4$ & $25.3 \pm 3.0$ & $<29.9$ & $44.9 \pm 11.5$ & $<111.0$ & $\ldots$ & $\ldots$ & $\ldots$ \\
\hline 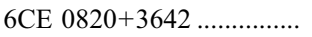 & $79.2 \pm 8.1$ & $81.9 \pm 8.4$ & $82.0 \pm 8.4$ & $68.0 \pm 7.0$ & $\ldots$ & $\ldots$ & $\ldots$ & $\ldots$ \\
\hline 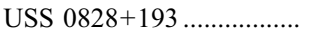 & $61.7 \pm 6.9$ & $133.0 \pm 13.0$ & $201.0 \pm 21.0$ & $687.0 \pm 74.0$ & $1270.0 \pm 112.8$ & $\ldots$ & $\ldots$ & $\ldots$ \\
\hline 5C $7.269 \ldots \ldots \ldots \ldots \ldots \ldots \ldots \ldots . .$. & $41.0 \pm 4.5$ & $49.5 \pm 5.3$ & $57.8 \pm 6.1$ & $<40.1$ & $<186.0$ & $\ldots$ & $\cdots$ & $\cdots$ \\
\hline 6CE $0901+3551 \ldots \ldots \ldots \ldots \ldots . .$. & $37.2 \pm 4.1$ & $46.5 \pm 5.0$ & $52.8 \pm 10.7$ & $69.8 \pm 12.4$ & $\ldots$ & $\ldots$ & $\ldots$ & $\ldots$ \\
\hline 6CE $0905+3955 \ldots \ldots \ldots \ldots \ldots . . .$. & $51.8 \pm 5.4$ & $60.1 \pm 6.2$ & $96.8 \pm 9.8$ & $146.0 \pm 14.0$ & $\ldots$ & $\ldots$ & $\ldots$ & $\ldots$ \\
\hline B2 $0902+34 \ldots \ldots \ldots \ldots \ldots \ldots$ & $6.4 \pm 0.8$ & $9.9 \pm 1.1$ & $11.0 \pm 2.5$ & $41.3 \pm 2.3$ & $144.0 \pm 39.6$ & $323.0 \pm 18.8$ & $\ldots$ & $<18500$ \\
\hline TN J0924-2201 ….............. & $11.3 \pm 1.8$ & $11.4 \pm 1.9$ & $<30.6$ & $<33.0$ & $<108.0$ & $<160.0$ & $<3330$ & $<52900$ \\
\hline 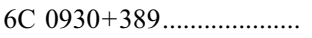 & $30.7 \pm 3.4$ & $32.2 \pm 3.6$ & $37.3 \pm 9.2$ & $<32.6$ & $<97.2$ & $\ldots$ & $\ldots$ & $\ldots$ \\
\hline 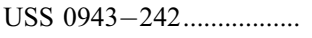 & $21.5 \pm 2.6$ & $28.4 \pm 3.2$ & $<30.9$ & $25.8 \pm 11.7$ & $109.0 \pm 34.1$ & $493.0 \pm 57.5$ & $<3390$ & $<50900$ \\
\hline 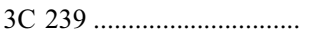 & $96.4 \pm 9.8$ & $111.0 \pm 11.0$ & $130.0 \pm 12.0$ & $142.0 \pm 14.0$ & $\ldots$ & $\ldots$ & $\ldots$ & $\ldots$ \\
\hline MRC 1017-220 .............. & $119.0 \pm 12.0$ & $179.0 \pm 18.0$ & $273.0 \pm 27.0$ & $360.0 \pm 36.0$ & $\ldots$ & $\ldots$ & $\ldots$ & $\ldots$ \\
\hline 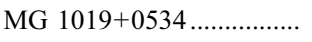 & $25.6 \pm 2.9$ & $19.5 \pm 3.8$ & $<35.4$ & $<42.9$ & $161.0 \pm 52.5$ & $\cdots$ & $\cdots$ & $\cdots$ \\
\hline WN J1115+5016 …................. & $7.8 \pm 2.1$ & $9.5 \pm 3.0$ & $<54.8$ & $<61.1$ & $<88.0$ & $\ldots$ & $\ldots$ & $\ldots$ \\
\hline 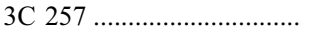 & $85.0 \pm 8.7$ & $111.0 \pm 11.0$ & $194.0 \pm 19.0$ & $322.0 \pm 33.0$ & $569.0 \pm 74.6$ & $\ldots$ & $\ldots$ & $\ldots$ \\
\hline WN J1123+3141 …........... & $48.2 \pm 5.0$ & $74.4 \pm 7.6$ & $92.7 \pm 9.4$ & $182.6 \pm 18.4$ & $742.0 \pm 70.4$ & $\ldots$ & $\ldots$ & $\ldots$ \\
\hline 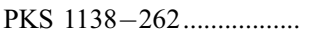 & $318.0 \pm 32.0$ & $497.0 \pm 50.0$ & $887.0 \pm 89.0$ & $1500.0 \pm 150.0$ & $2000.0 \pm 160.3$ & $3890.0 \pm 176.2$ & $\ldots$ & $\cdots$ \\
\hline 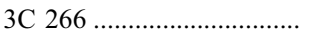 & $67.9 \pm 7.0$ & $73.1 \pm 7.5$ & $45.1 \pm 4.7$ & $102.6 \pm 10.4$ & $\ldots$ & $\ldots$ & $\ldots$ & $\cdots$ \\
\hline $6 \mathrm{C} 1232+39 \ldots \ldots \ldots \ldots \ldots \ldots \ldots$ & $33.3 \pm 3.6$ & $41.8 \pm 4.4$ & $52.0 \pm 9.2$ & $75.2 \pm 10.8$ & $208.0 \pm 35.9$ & $\ldots$ & $\ldots$ & $\ldots$ \\
\hline 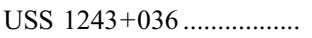 & $22.0 \pm 2.5$ & $21.5 \pm 3.0$ & $<48.0$ & $<62.9$ & $<163.0$ & $\ldots$ & $\ldots$ & $\ldots$ \\
\hline TN J1338-1942 ............... & $17.8 \pm 1.9$ & $10.7 \pm 1.2$ & $14.3 \pm 2.8$ & $9.9 \pm 3.4$ & $<119.0$ & $\ldots$ & $\ldots$ & $\ldots$ \\
\hline 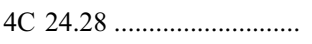 & $16.5 \pm 2.2$ & $27.3 \pm 3.1$ & $43.3 \pm 8.3$ & $102.0 \pm 10.0$ & $446.0 \pm 50.8$ & $\ldots$ & $\cdots$ & $\cdots$ \\
\hline 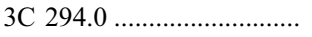 & $<93.0$ & $<103.0$ & $68.0 \pm 16.8$ & $66.6 \pm 20.6$ & $\ldots$ & $\ldots$ & $\ldots$ & $\ldots$ \\
\hline USS $1410-001 \ldots \ldots \ldots \ldots \ldots \ldots$ & $50.6 \pm 5.3$ & $79.0 \pm 8.1$ & $166.0 \pm 17.0$ & $240.0 \pm 24.0$ & $481.0 \pm 58.6$ & $\ldots$ & $\ldots$ & $\ldots$ \\
\hline $8 \mathrm{C} 1435+635 \ldots \ldots \ldots \ldots \ldots \ldots$ & $18.6 \pm 2.1$ & $14.7 \pm 2.9$ & $<45.5$ & $<50.3$ & $<86.7$ & $<165.0$ & $<4130$ & $<33900$ \\
\hline 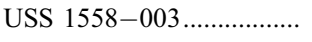 & $78.8 \pm 8.1$ & $101.0 \pm 10.3$ & $105.0 \pm 10.5$ & $233.0 \pm 23.4$ & $398.0 \pm 52.2$ & $\ldots$ & $\ldots$ & $\ldots$ \\
\hline 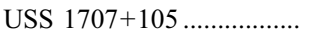 & $22.1 \pm 2.7$ & $30.1 \pm 3.4$ & $22.6 \pm 9.1$ & $<33.3$ & $<123.0$ & $\ldots$ & $\ldots$ & $\ldots$ \\
\hline LBDS 53w002 ..................... & $32.0 \pm 3.3$ & $44.0 \pm 4.5$ & $49.9 \pm 5.2$ & $103.0 \pm 11.0$ & $177.0 \pm 33.9$ & $648.0 \pm 61.4$ & $<4300$ & $<65100$ \\
\hline LBDS 53w091 ….................. & $43.3 \pm 6.3$ & $51.4 \pm 5.3$ & $23.9 \pm 6.1$ & $26.6 \pm 6.4$ & $\ldots$ & $<45.9$ & $<1610$ & $<23900$ \\
\hline 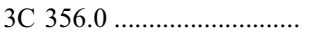 & $108.0 \pm 11.0$ & $110.0 \pm 11.0$ & $122.0 \pm 14.0$ & $434.0 \pm 47.0$ & $\ldots$ & $4060.0 \pm 192.3$ & $<4400$ & $<70200$ \\
\hline 7C $1751+6809 \ldots \ldots \ldots \ldots \ldots \ldots$ & $46.6 \pm 4.9$ & $50.8 \pm 5.3$ & $<40.9$ & $36.5 \pm 16.0$ & $\ldots$ & $351.0 \pm 50.5$ & $<3600$ & $<51600$ \\
\hline 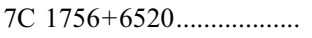 & $39.6 \pm 4.2$ & $46.9 \pm 5.0$ & $34.7 \pm 7.7$ & $42.6 \pm 8.6$ & $\ldots$ & $444.0 \pm 51.1$ & $<5900$ & $<151000$ \\
\hline 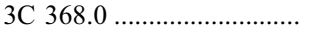 & $126.0 \pm 13.0$ & $112.0 \pm 11.0$ & $112.0 \pm 11.0$ & $210.0 \pm 21.0$ & $\ldots$ & $3250.0 \pm 166.7$ & $28800 \pm 2710$ & $<39000$ \\
\hline 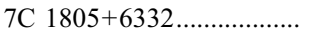 & $28.4 \pm 3.6$ & $42.1 \pm 4.4$ & $51.4 \pm 5.4$ & $95.6 \pm 17.1$ & $\ldots$ & $648.0 \pm 56.2$ & $<4330$ & $<111000$ \\
\hline 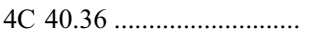 & $36.5 \pm 3.9$ & $41.3 \pm 4.3$ & $45.4 \pm 12.9$ & $26.3 \pm 10.3$ & $115.0 \pm 29.4$ & $520.0 \pm 55.2$ & $<4750$ & $<64500$ \\
\hline TX J1908+7220_................ & $200.0 \pm 20.0$ & $229.0 \pm 23.0$ & $241.0 \pm 25.0$ & $480.0 \pm 48.0$ & $841.0 \pm 70.6$ & $1910.0 \pm 98.9$ & $16200 \pm 1905$ & $<63300$ \\
\hline WN J1911+6342 „............. & $9.5 \pm 1.8$ & $8.7 \pm 1.8$ & $<26.4$ & $<26.0$ & $<75.3$ & $<142.0$ & $<3710$ & $<57100$ \\
\hline TN J2007-1316 ................. & $56.2 \pm 5.9$ & $54.4 \pm 5.8$ & $41.1 \pm 10.7$ & $121.3 \pm 12.3$ & $244.0 \pm 53.5$ & $\ldots$ & $\ldots$ & $\ldots$ \\
\hline 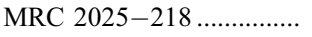 & $68.4 \pm 7.1$ & $77.1 \pm 8.0$ & $86.8 \pm 10.9$ & $126.8 \pm 12.9$ & $190.0 \pm 50.2$ & $\ldots$ & $\ldots$ & $\ldots$ \\
\hline 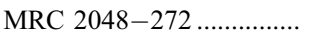 & $59.5 \pm 6.2$ & $72.6 \pm 7.5$ & $78.3 \pm 10.4$ & $38.4 \pm 13.1$ & $<141.0$ & $\cdots$ & $\cdots$ & $\cdots$ \\
\hline MRC 2104-242 ............... & $28.1 \pm 3.3$ & $29.7 \pm 3.5$ & $32.8 \pm 10.0$ & $<36.3$ & $121.0 \pm 47.0$ & $\ldots$ & $\ldots$ & $\ldots$ \\
\hline 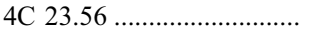 & $61.1 \pm 6.4$ & $86.2 \pm 8.8$ & $126.9 \pm 12.8$ & $423.7 \pm 42.5$ & $1610.0 \pm 129.8$ & $4390.0 \pm 203.8$ & $30300 \pm 2958$ & $<70500$ \\
\hline
\end{tabular}


TABLE 3-Continued

\begin{tabular}{|c|c|c|c|c|c|c|c|c|}
\hline HzRG & $f_{3.6 \mu \mathrm{m}}$ & $f_{4.5 \mu \mathrm{m}}$ & $f_{5.8 \mu \mathrm{m}}$ & $f_{8.0 \mu \mathrm{m}}$ & $f_{16 \mu \mathrm{m}}$ & $f_{24 \mu \mathrm{m}}$ & $f_{70 \mu \mathrm{m}}$ & $f_{160 \mu \mathrm{m}}$ \\
\hline 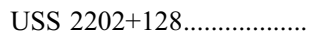 & $60.4 \pm 6.3$ & $95.8 \pm 9.8$ & $120.1 \pm 12.1$ & $106.6 \pm 10.8$ & $307.0 \pm 43.2$ & $\ldots$ & $\ldots$ & $\ldots$ \\
\hline B3 J2330+3927 .................... & $99.6 \pm 10.1$ & $143.0 \pm 14.0$ & $160.0 \pm 16.0$ & $474.0 \pm 47.0$ & $1040.0 \pm 88.0$ & $2210.0 \pm 113.2$ & $<4670$ & $<64300$ \\
\hline 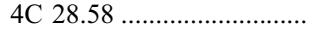 & $31.6 \pm 3.5$ & $36.0 \pm 3.9$ & $<41.7$ & $40.9 \pm 4.4$ & $228.0 \pm 31.5$ & & $\ldots$ & $\ldots$ \\
\hline 3C 470 & $49.5 \pm 10.4$ & $75.2 \pm 11.8$ & $70.9 \pm 10.4$ & $266.0 \pm 30.0$ & $\ldots$ & $2650.0 \pm 133.5$ & $<5570$ & $<102000$ \\
\hline
\end{tabular}

Figure 4 presents the measured mid-IR colors of the 49 HzRGs with detections in all four IRAC bands and the nine HzRGs with two-band IRAC detections on the color-color plots of Lacy et al. (2004) and Stern et al. (2005). As expected, the HzRG sample tends to fall within the AGN wedges identified in each of these diagrams. The Lacy et al. (2004) region includes nearly the entire HzRG sample: 51 out of 58 (88\%) of HzRGs plotted match the Lacy et al. (2007) color criteria (which are slightly updated from the criteria of Lacy et al. 2004). The smaller Stern et al. (2005) region misses a few more HzRGs, with only 41 out of 58 HzRGs (71\%) matching the Stern et al. (2005) color criteria. For both plots, nearly every HzRG is within $1 \sigma$ of matching the AGN color criteria.

It should be noted that, while the obscured and unobscured AGNs generally lie within the "AGN wedges" in Figure 4, these two classes of luminous AGN are presumed to have different energetics powering their observed mid-IR, IRAC-band SEDs. Luminous, unobscured AGNs tend to have power-law SEDs due to the central engine across the mid-IR regime (e.g., Richards et al. 2006). This leads to rising spectra through the IRAC passbands and places such sources squarely in the wedges at most redshifts. The caveat is that strong features can cause excursions as they redshift into the passbands; e.g., $\mathrm{H} \alpha$ at $z \approx 4$ is in the $3.6 \mu \mathrm{m}$ band (e.g., C. Bian et al. 2007, in preparation). In contrast to this, we presume that starlight plays a dominant role in the shorter wavelength IRAC passbands for most obscured AGNs, with AGN-heated dust dominating the reddest IRAC passbands. Indeed, a clear dip between the stellar component and the hot dust component is seen in many sources in our sample. Consider, as an example, the left-hand panel of Figure 3 . The redshifted galaxy tracks have [3.6] $-[4.5]>0.2$ (Vega) at $z \gtrsim 1$ due to the $1.6 \mu \mathrm{m}$ peak of the stellar SED redshifting into the $4.5 \mu \mathrm{m}$ IRAC passband. The red [3.6] - [4.5] colors are then usually due to the $z>1$ stellar populations, while the red [5.8] - [8.0] colors are due to the AGN. The next section will concern using the longer wavelength Spitzer data to model and subtract the AGN contribution to the mid-IR SEDs of our sample, and thus to derive the rest-frame near-IR stellar luminosities.

\section{MODELING OF THE REST-FRAME NEAR-INFRARED AND MID-INFRARED}

We begin our analysis of the broadband IR SEDs by considering the galaxies with the most extensive Spitzer coverage. This smaller sample of 26 galaxies illustrates the shape and range of HzRG SEDs in the IR ( $(4.2)$ and is then used to inform analysis of the sources with more restricted Spitzer imaging ( $(4.3)$.

Despite the extensive near-IR data available for many of the HzRGs, we elect not to include these data in the SED fitting (although the $K$-band photometry is overplotted in the SED plots).
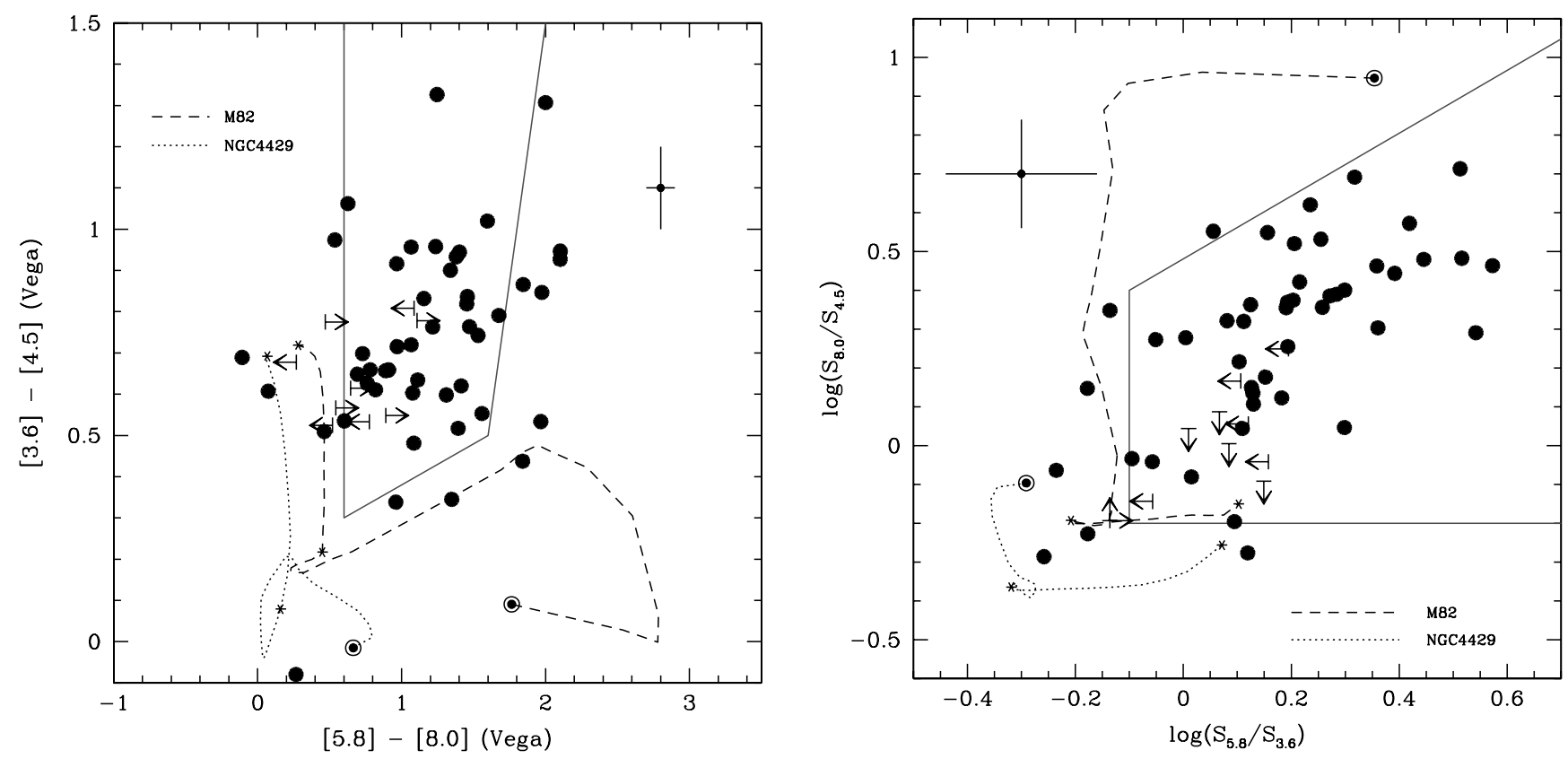

FIG. 4.-Mid-IR colors of HzRGs. Solid lines indicate empirical criteria that separate (type 1) active galaxies from Galactic stars and normal galaxies. The left panel

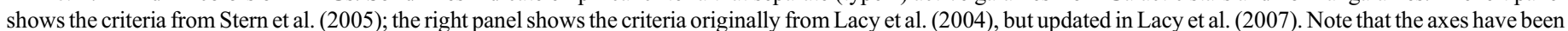

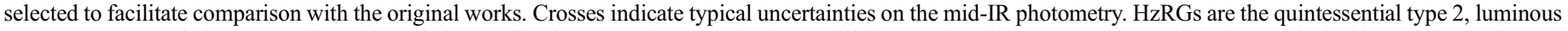

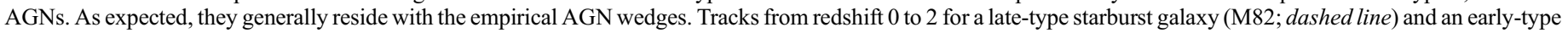

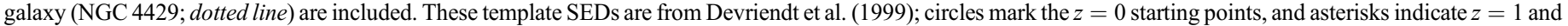

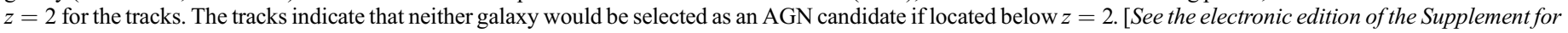
a color version of this figure.] 

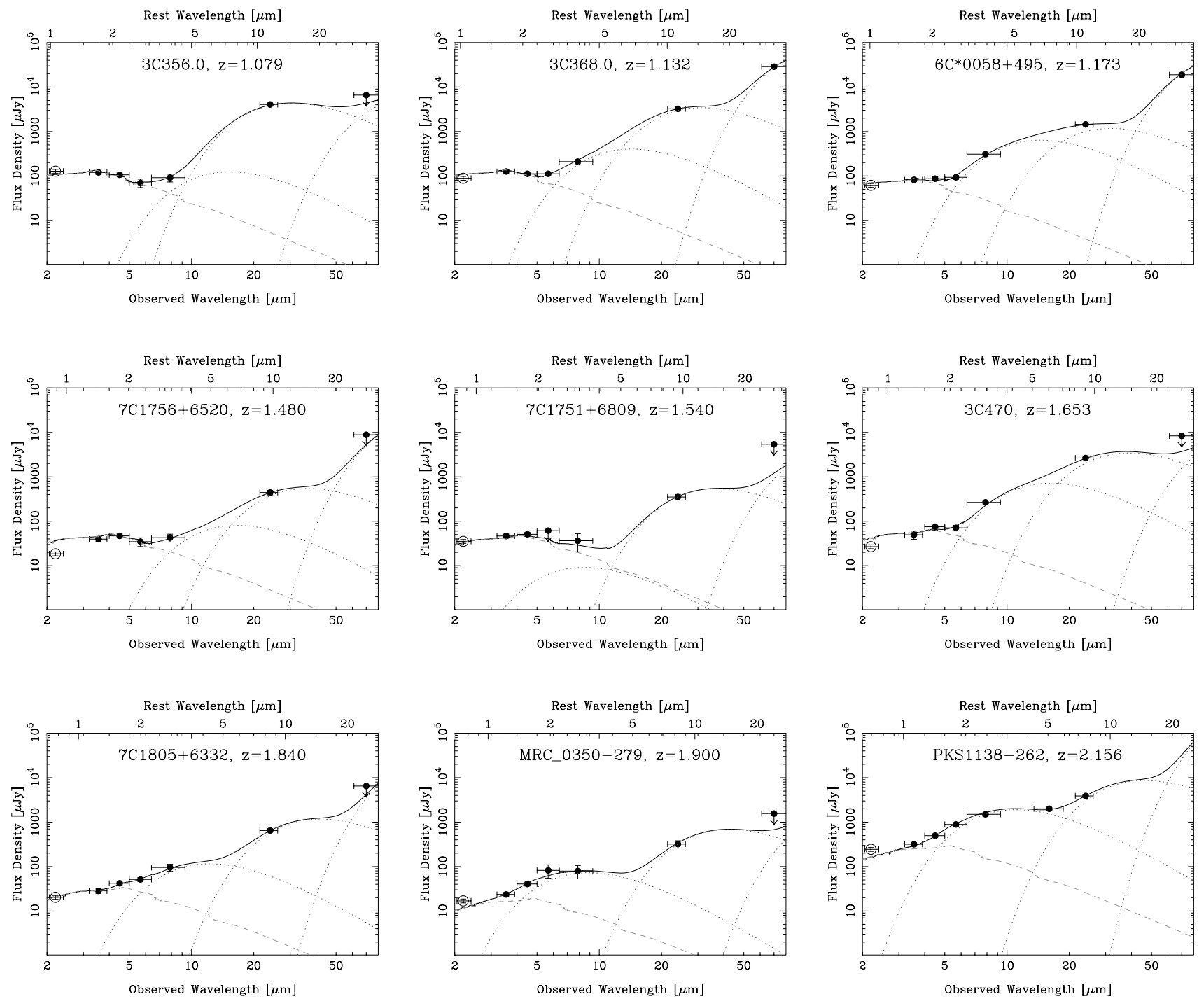

FIG. 5.- SED fitting of 21 sources with both IRAC observations and MIPS detections. The name of each HzRG and its redshift are marked at the top of the panel. Observed wavelength is along the bottom axis and rest-frame wavelength is along the top axis. Data with error bars are presented as filled circles; open circles are not used in the fitting for reasons described in the text. Downward arrows indicate upper limits. The solid dark line indicates the total best-fit SED. The stellar and dust components are indicated by dashed and dotted lines, respectively. [See the electronic edition of the Supplement for a color version of this figure.]

This omission has several reasons and, as described in $\S 5$, should have minimal impact on the derived stellar masses, the primary goal of our analysis. Foremost, we omit the near-IR data as it samples the rest-frame UV to optical wavelengths of our high-redshift galaxies. At these wavelengths, several issues arise. First, the scattered and direct AGN continuum is blue in color and will thus be a larger contributor to the SED at wavelengths below $4000 \AA$ (e.g., Vernet et al. 2001). Second, high equivalent width emission lines can significantly affect the near-IR photometry for certain redshifts (e.g., Villar-Martín et al. 2006). Indeed, when $\mathrm{H} \alpha$ is in the bluest IRAC channels (for our highest redshift sources), we do not include the affected channel in the SED modeling. Third, possible contributions from younger stellar populations will be most pronounced at shorter wavelengths. Fourth, rest-frame optical and UV data are more susceptible to dust extinction than rest-frame near-IR data. The final reason to omit the near-IR data is the large inhomogeneity of the data quality, depth, and analysis techniques. We are generally relying on published photometry done on $3 \mathrm{~m}$ to $10 \mathrm{~m}$ telescopes over the past two decades and extracted with a range of aperture sizes. Only in a few cases are we able to obtain the images from telescope archives.
For the sake of homogeneity of the analysis, the SED modeling described below relies solely on Spitzer photometry. In a forthcoming paper, we study the detailed stellar population properties of the HzRG sample, combining Spitzer data with archival data and data from our ongoing observing campaigns at Palomar Observatory and the VLT (A. Rettura et al. 2007, in preparation). For a few galaxies, we have already performed the full analysis using all available (optical to mid-IR) data (e.g., Villar-Martín et al. 2006; Stern et al. 2006; C. De Breuck et al. 2007, in preparation). As shown in $\S 5$, the derived stellar masses are consistent with the results of the simpler analysis presented here.

\subsection{The Model}

Twenty-six of our radio galaxies have both IRAC and MIPS observations, of which only four are not detected at $24 \mu \mathrm{m}$ (or in the longer wavelength MIPS channels). These four sources include three of the highest redshift radio galaxies and one radio galaxy at $z \sim 1.5$. In the latter case, this nondetection may be indicative of silicon absorption at rest-frame $9.8 \mu \mathrm{m}$. Alternatively, the undetected $z \sim 1.5$ radio galaxy, LBDS 53W091, is a low-luminosity radio source, and Stern et al. (2006) argue that its 

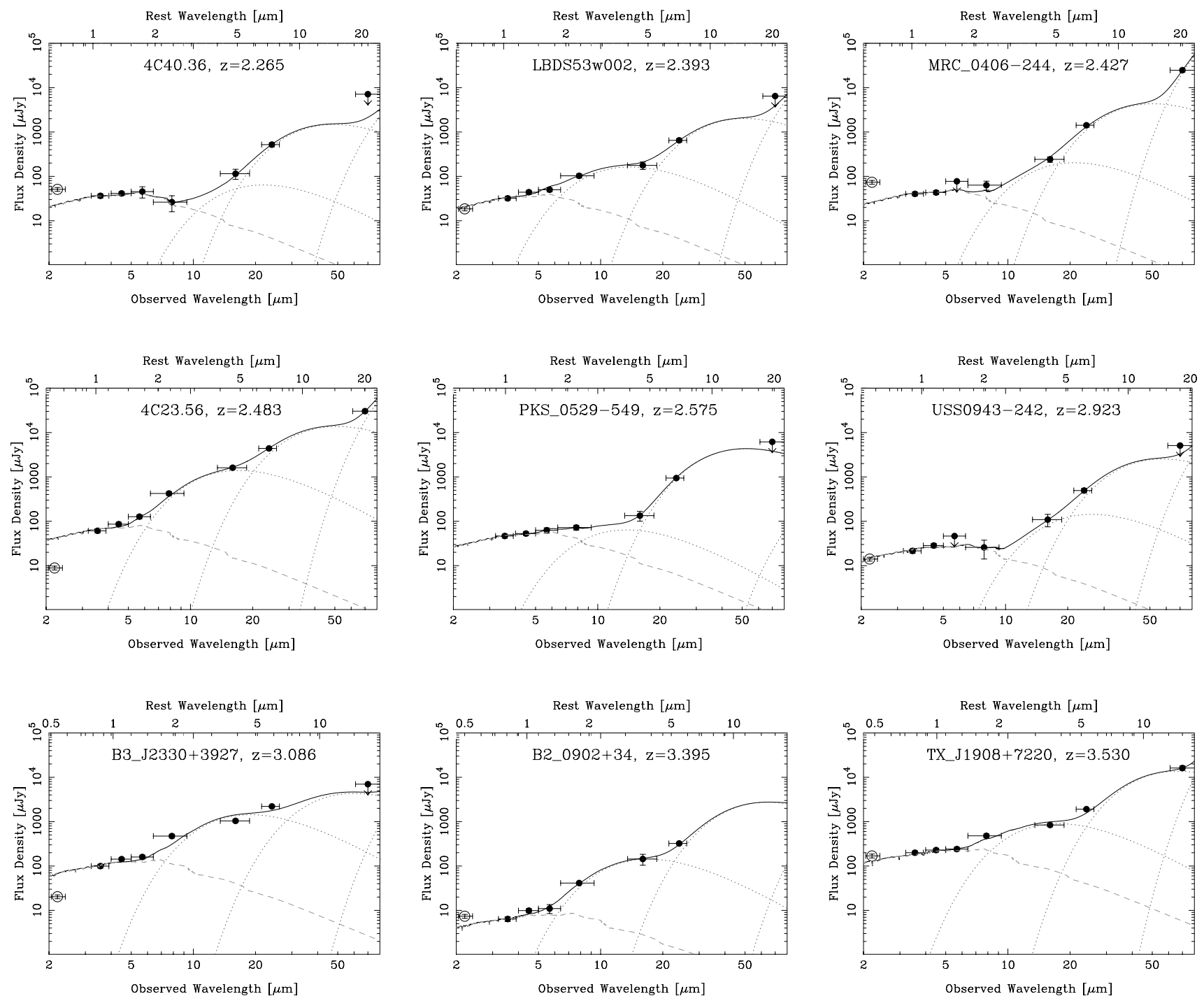

FIG. 5-Continued

mid-IR nondetection likely indicates a SED dominated by old stellar populations and not by hot, AGN-heated dust. One source, 6C $0140+326$, cannot be distinguished from a bright foreground galaxy at 3.6 and $4.5 \mu \mathrm{m}$, so we do not use it in our modeling here. Hence, our final sample numbers $21 \mathrm{HzRGs}$ with IRAC and MIPS detections.

For the 21 HzRGs with MIPS detections, we fit the SEDs with toy models comprised of a composite stellar population (CSP) and multiple blackbody (BB) dust components. Our primary goal is to simply derive the rest-frame near-IR stellar luminosity, which can then be translated into a stellar mass assuming reasonable stellar population synthesis models $(\S 5)$. We are also, in these cases with MIPS detections, able to estimate the rest-frame $5 \mu \mathrm{m}$ monochromatic luminosities, a possible measure of the obscured AGN power (Ogle et al. 2006). The difficulty is that in many sources a steeply rising continuum is visible in the redder IRAC bands, indicative of the tail of hot, AGN-heated dust contaminating the rest-frame near-IR emission. Owing to the sparse sampling of the SEDs at these wavelengths, our goal is not to fully explore the astrophysics of the continuum emission at these wavelengths, but to use the MIPS (and IRS, where available) photometry to model the dust SED and subtract its contribution at rest-frame near-IR wavelengths. We do not apply Galactic extinction cor- rections as they are negligible $(<0.5 \%)$ at the Spitzer wavelengths. Also, since emission lines longward of $\mathrm{H} \alpha$ are not strong enough to significantly affect our modeling (e.g., Haas et al. 2005; Ogle et al. 2006; Cleary et al. 2007), they are ignored in the analysis. Additional systematic issues in our modeling are discussed in $\S \S 4.4$ and 6.3 , but we stress here that the stellar masses are very insensitive to the choice of BBs for the longer wavelength component.

We model the radio galaxy IR SEDs with a four-component model consisting of an elliptical galaxy CSP template and three BBs of different dust temperatures. Best-fit parameters for the models are determined using standard $\chi^{2}$ minimization techniques. The uncertainties on the flux densities used to determine the $\chi^{2}$ of a specific fit include both random (from the observations) and systematic (e.g., the detector zero points and the shape of the SED) uncertainties, which are added in quadrature (see $\S 3$ ). The full fitting is only done for sources with MIPS $24 \mu \mathrm{m}$ detections as we need both enough data points to fit our model and a detection longward of the IRAC bands to provide sufficient leverage on the dust component (see $\S 4.2$ ). For sources without MIPS detections or observations we do not have enough data points to perform a secure fit of this four-component model. Hence, in many cases we only get an upper limit to the stellar component, although 

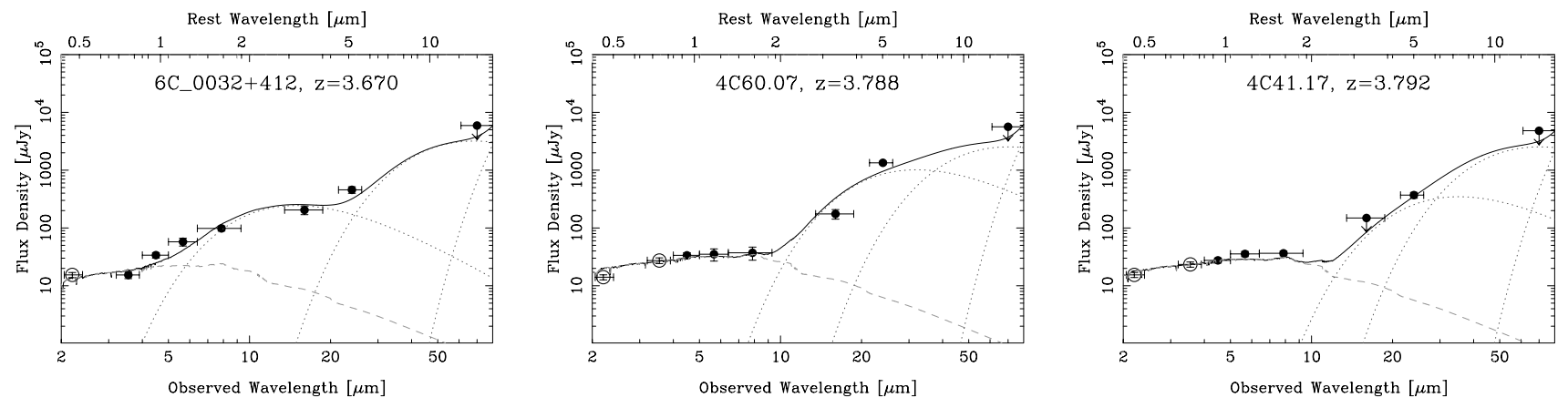

FIG. 5-Continued

in some cases we can get a likely value for the stellar luminosity when the IRAC bands fit the stellar component well (see $\S 4.3$ ).

\subsubsection{Stellar Component}

The CSP template used in our SED modeling consists of an elliptical galaxy modeled with PÉGASE.2 (Fioc \& Rocca-Volmerange 1997). The adopted star formation history (SFH) scenario has been shown to result in reliable photometric redshifts with the code Z-PEG (Le Borgne \& Rocca-Volmerange 2002) and to well reproduce early-type galaxy SEDs (from rest-frame UV to near-IR) up to $z \sim 1.25$ (Rettura et al. 2006). Specifically, the star-formation rate is proportional to the gas density, SFR $\propto \rho_{\text {gas }}$, and the astration (recycling) rate is equal to $3.33 \mathrm{Gyr}^{-1}$. Gaseous exchanges with the interstellar medium are represented by infall and galactic winds. The former process simulates mass growth, and the latter represses any star-formation after the first Gyr by dissipating the gas from the galaxy and hence driving the gas fraction toward zero. The reader is referred to the aforementioned papers for more details on the adopted template parameters and the starformation histories. In addition, Rocca-Volmerange et al. (2004) demonstrates how this elliptical galaxy model works for the most massive galaxies that populate the lower envelope of the $K-z$ relation. For the current work, we use a Kroupa (2001) initial mass function (IMF) and dust-free model templates. We note that in the templates used here, the metallicity is not a free parameter; it evolves consistently with the SFH, reaching solar values at the ages of interest in this study. We assume a galaxy formation redshift of $z_{\text {form }}=10$, and we discuss the systematic effects of this choice, along with the other details of the galaxy models in $\S 6.3$. In brief, however, although the derived stellar masses vary somewhat with the model choices (mainly by a simple offset depending on the choice of IMF), we find that the derived luminosities are relatively robust against model uncertainties for stellar population ages greater than $1 \mathrm{Gyr}$.

\subsubsection{Dust Component}

The longer wavelength modeling assumes three dust components, each of a different temperature. The coldest of the BBs is chosen to have a temperature $60 \mathrm{~K}$, representative of the large cool dust reservoirs associated with radio galaxies (e.g., Greve et al. 2006), although this component does not contribute to the restframe near-IR. The second BB component has a fixed temperature of $250 \mathrm{~K}$. For the final, hotter BB component we allowed the temperature to vary from 500 to $1500 \mathrm{~K}$ (in steps of $50 \mathrm{~K}$ ) and interpret it as being associated with hot dust heated by the central AGN. This upper limit of $1500 \mathrm{~K}$ was chosen as dust sublimates at this temperature. Systematic effects from the chosen BB temperatures are discussed in $\S 6.3$; in brief, we find they have little

TABLE 4

Results of SED Fitting for HzRGs with MIPS Detections

\begin{tabular}{|c|c|c|c|c|c|}
\hline HzRG & $\log \left(L_{\mathrm{H}}^{\mathrm{tot}} / L_{\odot}\right)$ & $\log \left(L_{\mathrm{H}}^{\text {stel }} / L_{\odot}\right)$ & $f_{\text {stel }}$ & $\log \left(M^{\text {stel }} / M_{\odot}\right)$ & $\log \left(L_{5 \mu \mathrm{m}} / L_{\odot}\right)$ \\
\hline $6 \mathrm{C} 0032+412 \ldots \ldots$ & 12.13 & $11.47_{-0.12}^{+0.16}$ & 0.22 & $11.28_{-0.12}^{+0.16}$ & $11.97_{-0.24}^{+0.56}$ \\
\hline $6 \mathrm{C}^{*} 0058+495 \ldots \ldots \ldots \ldots \ldots \ldots \ldots$ & 11.16 & $11.16_{-0.10}^{+0.13}$ & 0.99 & $11.26_{-0.10}^{+0.13}$ & $11.40_{-0.11}^{+0.16}$ \\
\hline 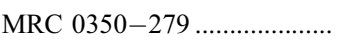 & 11.29 & $10.89_{-0.17}^{+0.26}$ & 0.40 & $10.85_{-0.17}^{+0.10}$ & $10.91_{-0.23}^{+0.11}$ \\
\hline MRC 0406-244 ….................... & 11.50 & $11.49_{-0.13}^{+0.13}$ & 0.98 & $11.38_{-0.13}^{+0.13}$ & $11.75_{-0.10}^{+0.11}$ \\
\hline 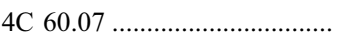 & 11.67 & $11.65_{-0.10}^{+0.13}$ & 0.95 & $11.44_{-0.10}^{+0.13}$ & $12.50_{-0.35}^{+0.35}$ \\
\hline PKS 0529-549 & 11.67 & $11.60_{-0.10}^{+0.11}$ & 0.86 & $11.46_{-0.10}^{+0.11}$ & $11.60_{-0.10}^{+0.14}$ \\
\hline 4C 41.17 & 11.60 & $11.60_{-0.08}^{+0.08}$ & 0.99 & $11.39_{-0.08}^{+0.08}$ & $12.06_{-0.36}^{+0.36}$ \\
\hline 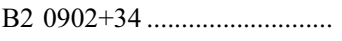 & 11.52 & $10.97_{-0.11}^{+0.14}$ & 0.28 & $10.81_{-0.11}^{+0.14}$ & $11.80_{-0.12}^{+0.10}$ \\
\hline 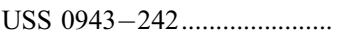 & 11.40 & $11.40_{-0.07}^{+0.15}$ & 0.99 & $11.22_{-0.07}^{+0.15}$ & $11.67_{-0.20}^{+0.35}$ \\
\hline PKS $1138-262 \ldots \ldots \ldots \ldots \ldots \ldots$ & 12.59 & $<12.18$ & $<0.39$ & $<12.11$ & $12.39_{-0.20}^{+0.35}$ \\
\hline 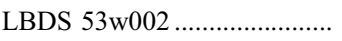 & 11.55 & $11.38_{-0.10}^{+0.12}$ & 0.68 & $11.27_{-0.10}^{+0.12}$ & $11.55_{-0.14}^{+0.22}$ \\
\hline 3С 356.0 & 11.28 & $11.28_{-0.10}^{+0.12}$ & 1.00 & $11.39_{-0.10}^{+0.12}$ & $11.39_{-0.07}^{+0.08}$ \\
\hline 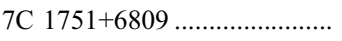 & 11.14 & $11.12_{-0.13}^{+0.13}$ & 0.95 & $11.14_{-0.13}^{+0.13}$ & $10.39_{-0.18}^{+0.30}$ \\
\hline 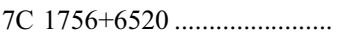 & 11.12 & $11.12_{-0.10}^{+0.12}$ & 1.00 & $11.15_{-0.10}^{+0.12}$ & $10.80_{-0.17}^{+0.27}$ \\
\hline 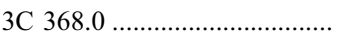 & 11.32 & $11.32_{-0.09}^{+0.11}$ & 0.99 & $11.43_{-0.09}^{+0.11}$ & $11.28_{-0.08}^{+0.10}$ \\
\hline 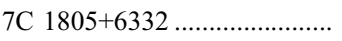 & 11.22 & $11.11_{-0.11}^{+0.15}$ & 0.78 & $11.07_{-0.11}^{+0.15}$ & $11.19_{-0.13}^{+0.17}$ \\
\hline $4 C 40.36$ & 11.38 & $11.38_{-0.10}^{+0.13}$ & 1.00 & $11.29_{-0.10}^{+0.13}$ & $11.21_{-0.15}^{+0.23}$ \\
\hline TX J1908+7220 ….................... & 12.63 & $<12.44$ & $<0.64$ & $<12.27$ & $12.60_{-0.11}^{+0.13}$ \\
\hline 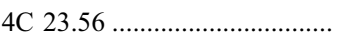 & 11.91 & $11.71_{-0.09}^{+0.12}$ & 0.63 & $11.59_{-0.09}^{+0.12}$ & $12.49_{-0.09}^{+0.10}$ \\
\hline 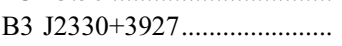 & 12.30 & $<12.11$ & $<0.64$ & $<11.94$ & $12.58_{-0.16}^{+0.26}$ \\
\hline 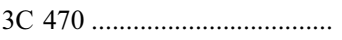 & 11.32 & $11.30_{-0.15}^{+0.21}$ & 0.95 & $11.30_{-0.15}^{+0.21}$ & $11.80_{-0.09}^{+0.11}$ \\
\hline
\end{tabular}


effect on the derived stellar masses. We have also experimented with toroidal AGN models (e.g., Pier \& Krolik 1992; Granato et al. 1997; Nenkova et al. 2002; Siebenmorgen et al. 2004). However, the paucity of our infrared data, consisting simply of photometric points and not infrared spectra, makes fitting with detailed models ill-constrained. We emphasize that this dust modeling is not completely physical; however, the modeling is sufficient for the primary goal of this paper, which is to simply estimate and subtract any contribution by (hot) dust to the rest-frame near-IR emission.

\subsection{Fits to HzRGs with MIPS Detections}

The best-fit models for the 21 HzRGS with MIPS detections are shown in Figure 5. Observed wavelength is indicated along the bottom axis, and rest wavelength is on the top axis. Open circles, not used in the fitting, are either (1) $K$-band data or (2) potentially contaminated by strong emission lines (e.g., H $\alpha$ ). Upper limits are indicated by downward arrows. We separate the contribution of host dust and stellar emission at rest-frame $H$ band, which can then be used to derive stellar masses ( $(5)$ ). Table 4 presents the total $H$-band luminosities, the stellar $H$-band luminosities, the stellar fractions at $H$ band, stellar masses, and restframe $5 \mu \mathrm{m}$ monochromatic luminosities for sources with full SED fitting. This fiducial mid-IR wavelength, $5 \mu \mathrm{m}$, was selected as it is always shortward of the $24 \mu \mathrm{m}$ MIPS observations, even for our most distant HzRGs with $24 \mu$ m detections $(z \sim 3.8)$. The formal $\chi^{2}$ uncertainties $(95.4 \%$ confidence limits) of the stellar luminosities from the fitting are listed in Table 4 . They are generally of the order $\sim 25 \%$ and are mainly due to the uncertainty in the IRAC flux densities, which, in turn, are dominated by systematics.

\subsection{Fits to HzRGs without MIPS Observations or Detections}

We can constrain, or in some cases quantify, the stellar luminosities for sources without MIPS observations or detections using the results of the full SED fitting described above (see Table 5). For many HzRGs we clearly observe the $H$-band stellar peak (see Fig. 5). Even in those cases where we do not see the $H$-band peak, IRAC channels 1 and 2 are typically dominated by the stellar component. At the least, we can obtain an upper limit to the stellar mass by demanding that the stellar component does not exceed any of the IRAC photometric points. Hence, we can obtain upper limits to the stellar masses of these HzRGs. Typically, this upper limit is restricted by just IRAC channel 1, as the observed mid-IR SEDs are often quite red. In many cases, the maximum fit is constrained by IRAC channels 1 and 2, and occasionally by longer wavelength channels. In almost half of the radio galaxy sample, the stellar SED is well fitted in two or three channels (those at shorter wavelength), so we nominally fit the SED and derive stellar luminosities. The results of the fitting for the HzRGs without MIPS observations or detections are shown in Figure 6, which adopts the same symbol convention as Figure 5 bar the inclusion of BB dust components. As can be seen, the $16 \mu \mathrm{m}$ observations, where present, generally show a strong excess above the maximally fitted stellar template. In what follows, we distinguish those HzRGs whose IRAC SEDs are well fitted by a stellar population (e.g., where multiple IRAC channels are well fitted by the stellar SED model) from those where the IRAC photometry has merely provided an upper limit to the rest-frame near-IR stellar luminosity.

\subsection{Systematic Effects in the SED Modeling}

We discuss here two possible systematic effects in the SED modeling: the adopted BB temperatures and potential photometric offsets in the longer wavelength photometry due to strong
TABLE 5

Results of SED Fitting for HzRGs with no MIPS Detections

\begin{tabular}{|c|c|c|c|}
\hline HzRG & $\log \left(L_{\mathrm{H}}^{\mathrm{tot}} / L_{\odot}\right)$ & $\log \left(M_{\max }^{\text {stel }} / M_{\odot}\right)$ & $\log \left(L_{5 \mu \mathrm{m}} / L_{\odot}\right)$ \\
\hline MRC $0037-258 \ldots \ldots \ldots \ldots$. & $<11.56$ & $<11.70$ & $\ldots$ \\
\hline MRC 0114-211 .............. & $<11.37$ & $<11.40$ & $\ldots$ \\
\hline TN J0121+1320_............. & 11.21 & 11.00 & $\ldots$ \\
\hline $6 C^{*} 0132+330 \ldots \ldots \ldots \ldots \ldots$ & 11.11 & 11.10 & $\ldots$ \\
\hline $6 \mathrm{C} 0140+326 \ldots \ldots \ldots \ldots \ldots$ & $<11.78$ & $<11.40$ & $\ldots$ \\
\hline MRC $0152-209 \ldots \ldots \ldots \ldots . . .$. & $<11.72$ & $<11.70$ & $\ldots$ \\
\hline MRC $0156-252 \ldots \ldots \ldots \ldots . .$. & $<12.23$ & $<12.20$ & 12.21 \\
\hline TN J0205+2242............. & $<10.96$ & $<10.80$ & $\ldots$ \\
\hline MRC $0211-256 \ldots \ldots \ldots \ldots$. & $<11.54$ & $<11.60$ & 11.23 \\
\hline 3C 65 & 11.44 & 11.50 & $\ldots$ \\
\hline MRC $0251-273 \ldots \ldots \ldots \ldots . .$. & 11.14 & 11.00 & $\cdots$ \\
\hline MRC 0316-257 ............. & 11.39 & 11.20 & $\ldots$ \\
\hline MRC 0324-228 ............. & 11.21 & 11.20 & $\ldots$ \\
\hline WN J0617+5012 ............ & 10.74 & 10.60 & $\ldots$ \\
\hline WN J0747+3654 …........ & 11.34 & 11.20 & $\ldots$ \\
\hline 6CE $0820+3642 \ldots \ldots \ldots \ldots$. & 11.54 & 11.50 & $\ldots$ \\
\hline USS $0828+193 \ldots \ldots \ldots \ldots . .$. & $<11.72$ & $<11.60$ & 12.34 \\
\hline 5C 7.269 & 11.48 & 11.40 & $\ldots$ \\
\hline 6CE $0901+3551 \ldots \ldots \ldots . .$. & 11.32 & 11.30 & $\ldots$ \\
\hline 6CE 0905+3955 .............. & 11.40 & 11.40 & $\ldots$ \\
\hline TN J0924-2201 ............. & 11.48 & 11.10 & $<11.79$ \\
\hline $6 \mathrm{C} 0930+389 \ldots \ldots \ldots \ldots \ldots$ & 11.40 & 11.30 & $\ldots$ \\
\hline 3С 239 & 11.60 & 11.60 & $\ldots$ \\
\hline MRC 1017-220 ............. & $<11.74$ & $<11.70$ & $\ldots$ \\
\hline MG $1019+0534 \ldots \ldots \ldots \ldots$ & $<11.34$ & $<11.20$ & 11.50 \\
\hline WN J1115+5016 …........ & $<10.86$ & $<10.70$ & $\ldots$ \\
\hline 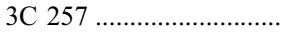 & $<11.83$ & $<11.70$ & 11.96 \\
\hline WN J1123+3141 ........... & $<11.81$ & $<11.60$ & $\ldots$ \\
\hline 3C 266 & 11.22 & 11.30 & $\ldots$ \\
\hline $6 \mathrm{C} 1232+39 \ldots \ldots \ldots \ldots \ldots \ldots$ & $<11.66$ & $<11.50$ & $\ldots$ \\
\hline USS $1243+036 \ldots \ldots \ldots \ldots . . .$. & $<11.47$ & $<11.30$ & $\ldots$ \\
\hline TN J1338-1942 …........ & $<11.26$ & $<11.00$ & $\ldots$ \\
\hline 4C 24.28 & $<11.20$ & $<11.00$ & $\ldots$ \\
\hline 3C $294.0 \ldots \ldots \ldots \ldots \ldots \ldots . .$. & $<11.50$ & $<11.50$ & $\ldots$ \\
\hline USS $1410-001 \ldots \ldots \ldots \ldots . . .$. & $<11.53$ & $<11.40$ & 11.86 \\
\hline $8 \mathrm{C} 1435+635 \ldots \ldots \ldots \ldots \ldots$ & $<11.45$ & $<11.10$ & $<11.63$ \\
\hline USS $1558-003 \ldots \ldots \ldots \ldots \ldots$ & 11.85 & 11.70 & 11.83 \\
\hline USS $1707+105 \ldots \ldots \ldots \ldots . .$. & 11.28 & 11.20 & $\ldots$ \\
\hline LBDS 53w091 ….............. & 11.20 & 11.20 & $<10.88$ \\
\hline WN J1911+6342 ........... & $<11.13$ & $<10.90$ & $<11.33$ \\
\hline TN J2007-1316 .............. & $<11.79$ & $<11.60$ & $\ldots$ \\
\hline MRC $2025-218 \ldots \ldots \ldots \ldots .$. & 11.80 & 11.60 & 11.53 \\
\hline MRC $2048-272 \ldots \ldots \ldots \ldots . .$. & $<11.55$ & $<11.50$ & $\ldots$ \\
\hline MRC $2104-242 \ldots \ldots \ldots \ldots . .$. & 11.34 & 11.20 & 11.30 \\
\hline MG 2144+1928 ............... & $<11.48$ & $<11.30$ & $\ldots$ \\
\hline USS $2202+128 \ldots \ldots \ldots \ldots . . .$. & $<11.85$ & $<11.70$ & 11.77 \\
\hline MRC 2224-273 …......... & $<11.34$ & $<11.30$ & $\ldots$ \\
\hline 4C 28.58 & $<11.56$ & $<11.40$ & 11.68 \\
\hline
\end{tabular}

spectral features redshifting into the Spitzer passbands. The latter effect mimics the effects of alternate SEDs with strong absorption and/or emission features. As seen below, neither systematic issue strongly affects the derived rest-frame near- and mid-IR luminosities.

As the cooler dust components have negligible influence at rest-frame $H$ band, the exact temperatures used are not vital to the final derived stellar luminosities and masses. Modeling observations of 3C radio galaxies obtained with the Infrared Space Observatory, Rocca-Volmerange \& Remazeilles (2005) find the far-IR SEDs are well fitted with two BB components of temperatures $40 \pm 16$ and $340 \pm 50 \mathrm{~K}$. Archibald et al. (2001) modeling submillimeter observations of HzRGs, likewise adopts an optically thin, isothermal gray-body of temperature $40 \mathrm{~K}$. When 

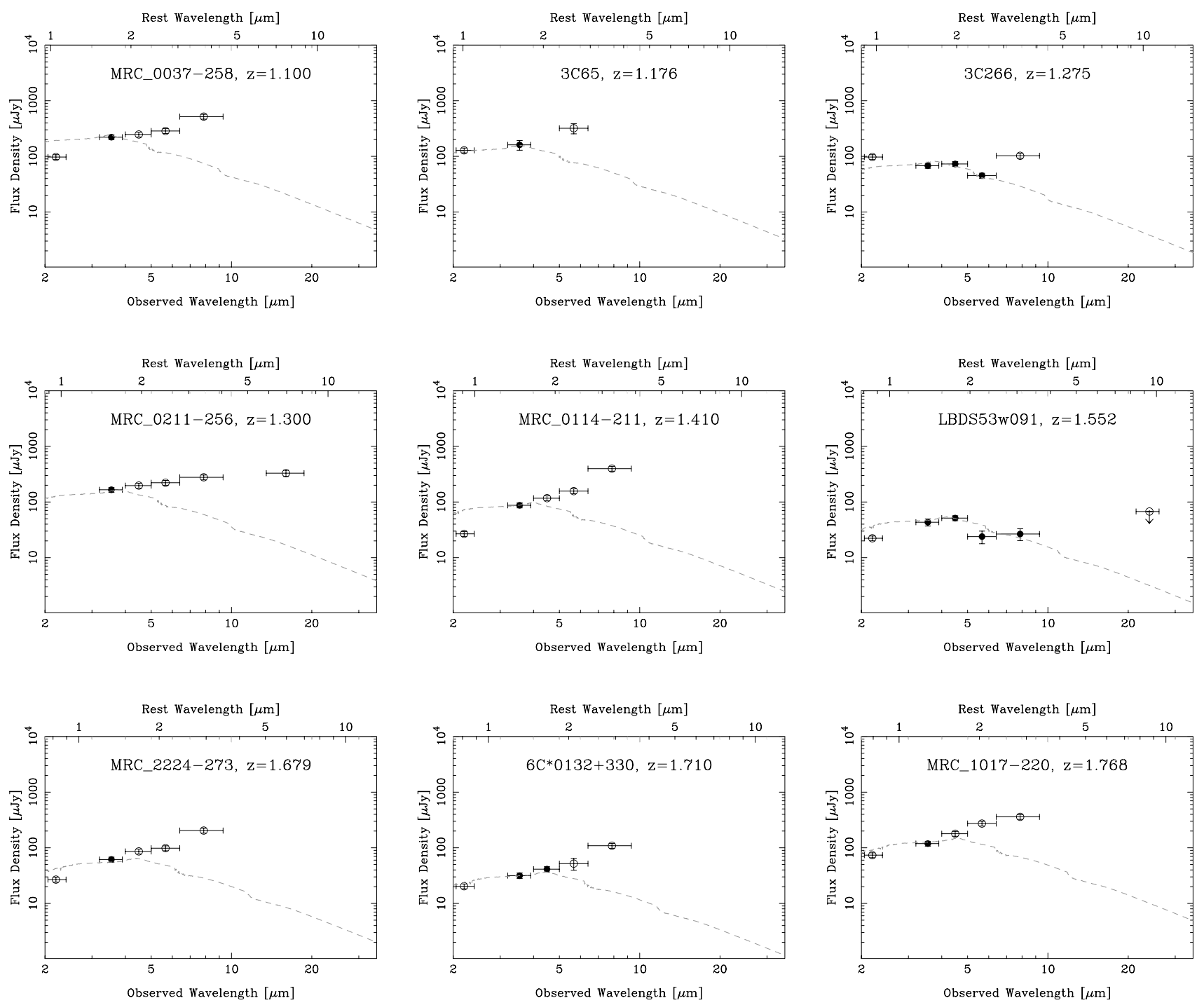

FIG. 6.-Galaxy SED fits to determine stellar masses or upper limits. The name of each HzRG and its redshift are marked at the top of the panel. Observed wavelength is along the bottom axis and rest-frame wavelength is along the top axis. Data with error bars are presented as filled circles; open circles not used in the fitting are described in the text. Downward arrows indicate upper limits. The dashed line indicates the total best-fit SED. [See the electronic edition of the Supplement for a color version of this figure.]

we redo our analysis adopting this cooler cold dust component rather than the previously adopted $60 \mathrm{~K}$, the derived rest-frame near/mid-IR luminosities change by less than $0.1 \%$. Likewise, using the $340 \mathrm{~K}$ warm dust component of Rocca-Volmerange \& Remazeilles (2005) instead of the previously adopted $250 \mathrm{~K}$, we find that the derived rest-frame near-IR luminosities change by less than $0.1 \%$, although the mid-IR luminosities increase by $\sim 40 \%$.

The fitting algorithm we adopt assumes a very simplistic model of the long-wavelength HzRG SED. No HzRGs have been observed to have PAH features (Ogle et al. 2006; Cleary et al. 2007), but some less luminous, radio-quiet type 2 AGNs have been seen with PAH features (M. Lacy et al. 2007, in preparation). Silicon absorption has been observed in HzRGs (Ogle et al. 2006), but even with a line width of $1.4 \mu \mathrm{m}$ and $\tau=1.5$ we would only observe a $30 \%-40 \%$ decrease in the observed flux density. Ogle et al. (2006) also note that there are indications that the powerlaw continuum is truncated at the sublimation temperature.

We have investigated the effects of these potential features by rerunning the fitting procedure, varying the 16 and $24 \mu \mathrm{m}$ flux densities to simulate those passbands falling on strong emission or absorption features. We varied the $16 \mu \mathrm{m}$ flux densities by
$10 \%$ to account for weaker PAH features at $6.4 \mu \mathrm{m}$ passing through the IRS bandpass. We varied the $24 \mu \mathrm{m}$ flux densities by $50 \%$ to account for both the strong $7.7 \mu \mathrm{m}$ PAH emission feature and the strong $9.8 \mu \mathrm{m}$ silicate absorption feature. These experiments only changed the mean $5 \mu$ m luminosities by $\sim 5 \%$ (for varying the $16 \mu \mathrm{m}$ flux density) and $\sim 25 \%$ (for varying the $24 \mu \mathrm{m}$ flux density). Given the lack of observational evidence for these features so far we consider these estimates conservative. The effects on the mean stellar luminosity (and hence mass) are $\$ 2 \%$ in both cases.

\section{RESULTS OF SED FITTING}

\subsection{Stellar Fractions}

Figures 7 and 8 show the fraction of the HzRG rest-frame $H$-band luminosity that is modeled as stellar in origin, plotted against total $H$-band luminosity, stellar $H$-band luminosity, redshift, radio power, and rest-frame $5 \mu \mathrm{m}$ luminosity. The stellar fraction is defined as the ratio of the flux densities found from convolving the stellar and total SEDs with a $H$-band filter curve.

Only the $21 \mathrm{HzRG}$ s with MIPS detections are presented in these figures and their $H$-band luminosities (stellar and total), stellar 

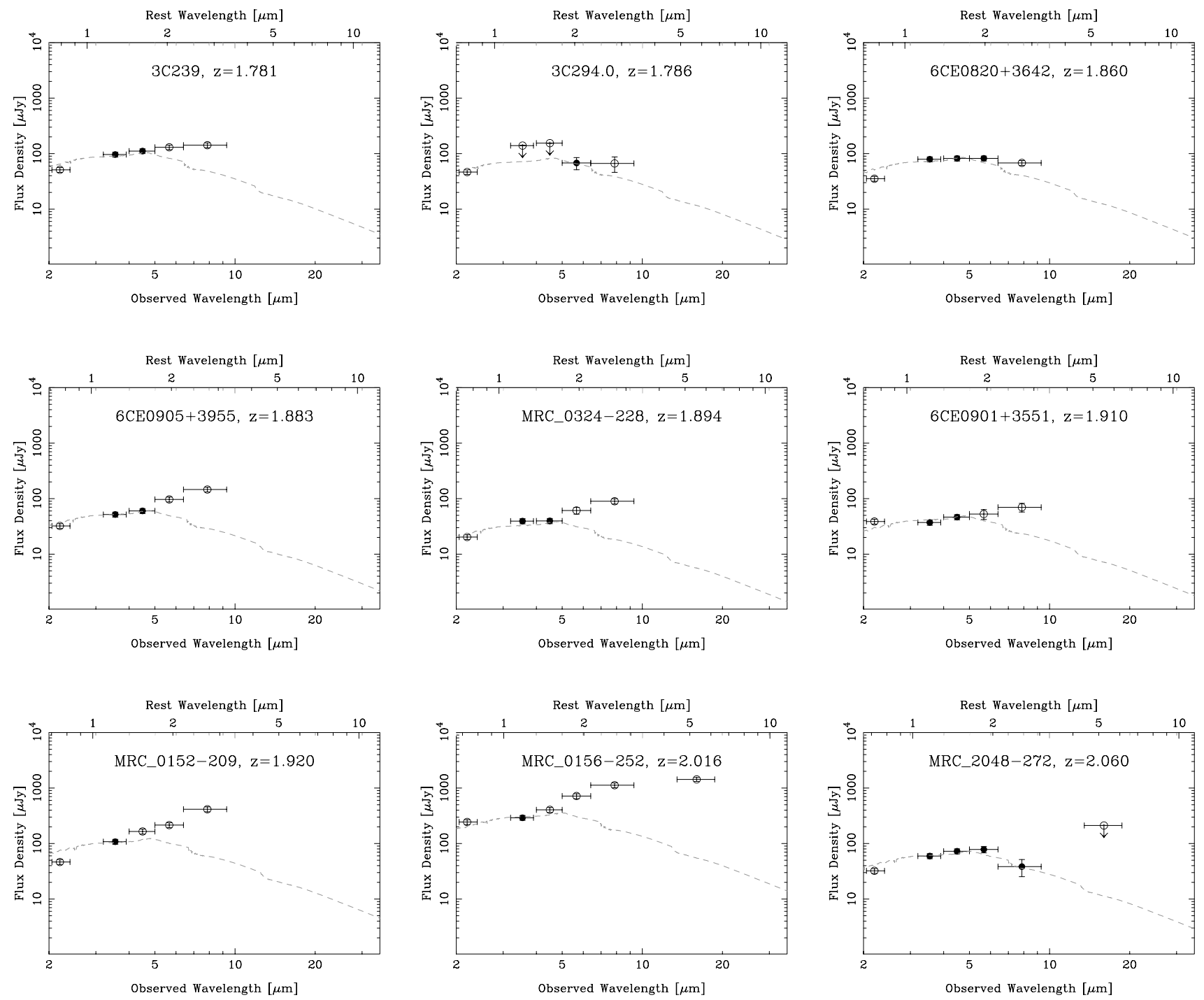

FIG. 6-Continued

fractions, inferred stellar masses (see $\S 6$ ), and monochromatic $5 \mu \mathrm{m}$ luminosities (see $\S 5.4$ ) in Table 4. As can be seen, half the sample has the rest-frame near-IR luminosity strongly dominated by starlight, with $\gtrsim 95 \%$ of the emission apparently stellar in origin. Another $20 \%$ of the sample have stellar emission accounting for $60 \%-90 \%$ of the rest-frame near-IR flux and thus still dominate the emission at this wavelength. There appears to be a weak inverse correlation between stellar fraction and total $H$-band luminosity, while the stellar fraction does not vary with stellar $H$-band luminosity. This suggests that the underlying host galaxy population is homogeneous. No evolutionary trends in the stellar fraction are evident, nor does the stellar fraction appear to correlate with either radio power or rest-frame mid-IR luminosity. This result is expected for the following reasons. The radio emission at rest-frame $3 \mathrm{GHz}$ is dominated by isotropic lobe emission. Likewise, rest-frame mid-IR samples largely isotropic emission coming from near the central engine (e.g., Heckman et al. 1994); at these wavelengths, the emission should be largely immune to extinction. The stellar fraction at $H$ band, however, is very dependent on obscuration of the AGNs, which, in the currently preferred unified schemes (e.g., Antonucci 1993; Urry \& Padovani 1995), is a function of orientation. AGNs with little obscuration of the central engine will have higher rest-frame near-IR luminosities, show broad lines, and have low stellar frac- tions. Indeed, the three sources in our MIPS sample with the highest rest-frame $H$-band luminosities, PKS $1138-262$, TX $\mathrm{J} 1908+7220$, and B3 J2330+3927, both show AGN signatures in the near-IR (see Appendix) and are indicated as upper limits in Table 4 and the relevant figures (Figs. 7 and 8). The stellar fraction is expected to be an indicator of orientation and should thus not correlate with the orientation-independent parameters. In a future publication (N. Seymour et al. 2007, in preparation), we shall analyze how the stellar fraction correlates with properties that are expected to depend on orientation, such as radio core fraction and radio spatial extent.

\subsection{Rest-Frame Near-IR Stellar Luminosities}

The rest-frame $H$-band stellar luminosities are found mainly to lie in the $10^{11}-10^{12} L_{\odot}$ range, as illustrated in Figure 9. Overlaid are PÉGASE models illustrating the luminosity of an elliptical galaxy model with $z_{\text {form }}=10$ for masses of $10^{11}$ and $10^{12} M_{\odot}$. The derived HzRG stellar luminosities are consistent with extremely massive hosts, with masses of a few $\times 10^{11} M_{\odot}$ out to $z=4$. Derivations of masses and their associated uncertainties are presented in $\S 6$.

This result confirms earlier work on the masses of radio galaxies. Rocca-Volmerange et al. (2004) find that most radio galaxies have $K$-band magnitudes consistent with stellar masses 

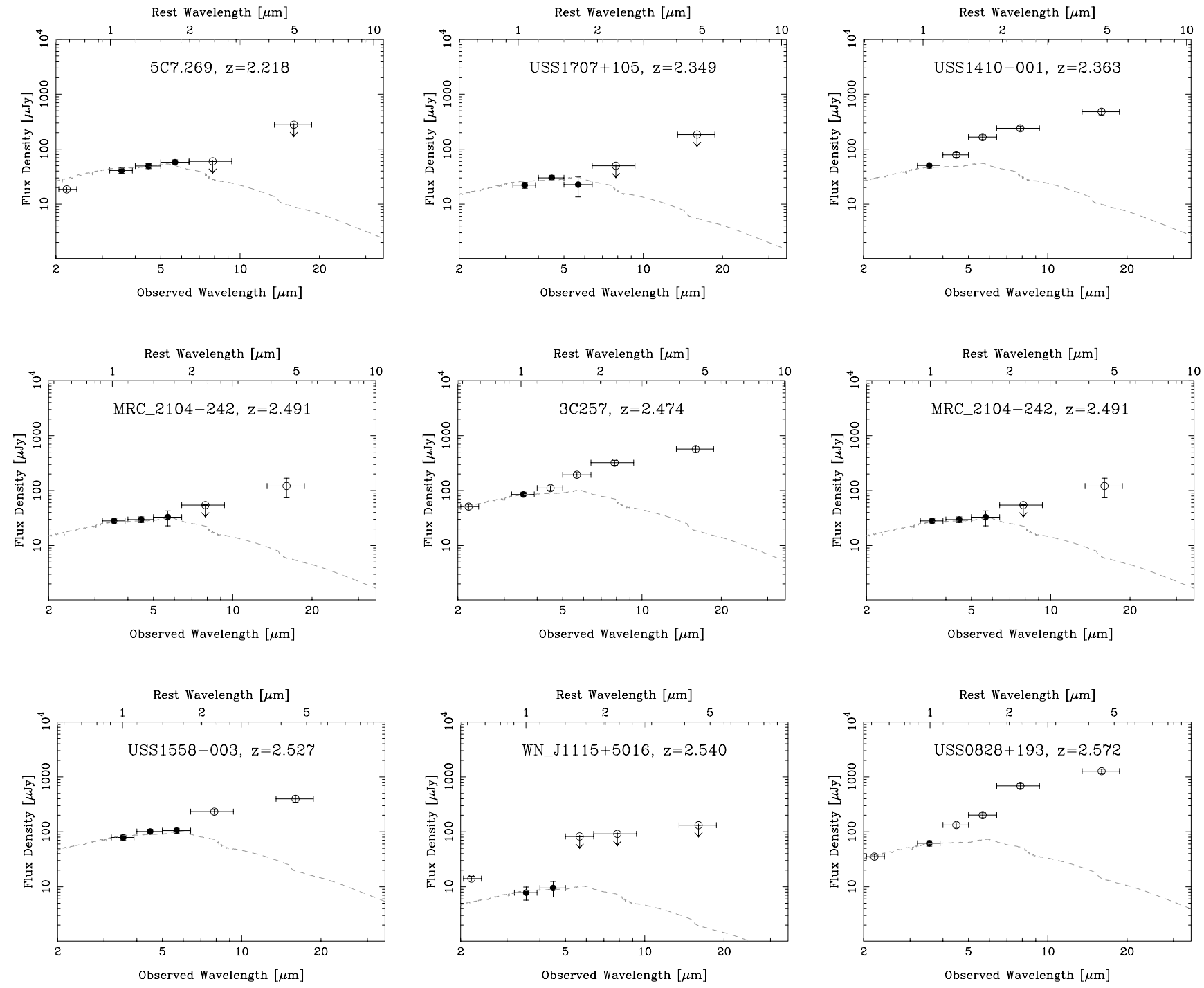

FIg. 6-Continued

of $10^{11}-10^{12} M_{\odot}$ with a few having magnitudes implying masses $>10^{12} M_{\odot}$. These sources with bright $K$-band magnitudes may have emission lines or scattered AGN light contributing to them, so their $K$-band magnitudes should be viewed as upper limits.

We also plot the rest $H$-band stellar luminosities of a sample of submillimeter galaxies (SMGs) in the GOODS North region from Borys et al. (2005) as red crosses in Figure 9. The $H$-band luminosities are rederived using our modeling technique with the data presented in Borys et al. (2005). Our analysis produces similar luminosities to Borys et al. (2005). However, due to different assumed star formation histories and IMFs, our derived stellar masses systematically differ by $25 \%$. These SMGs have a similar range of rest-frame $H$-band luminosities to the HzRGs, implying similar stellar masses. There is a probable evolutionary link between SMGs and HzRGs, although the number of similarities between these populations is comparable to the number of differences. On the one hand, SMGs have a much higher areal density than HzRGs: the former are relatively numerous in smallarea (100 $\left.\operatorname{arcmin}^{2}\right)$ surveys (e.g., Borys et al. 2003), while the latter are much rarer and typically herald from large-area or fullsky radio surveys. On the other hand, many radio galaxies are strong submillimeter emitters (e.g., Archibald et al. 2001; Greve et al. 2006) and would qualify as SMGs irrespective of their strong radio emission.

\subsection{Mid-IR Luminosities}

The rest-frame $5 \mu \mathrm{m}$ monochromatic luminosities presented in Table 4 are generally all above $10^{11} L \odot$. In Figure 10 we show the distribution of the $5 \mu \mathrm{m}$ luminosities with redshift. The solid symbols and arrows indicate the $21 \mathrm{HzRG}$ with MIPS detections and the five HzRGs with MIPS upper limits. The open symbols indicate the $13 \mathrm{HzRGs}$ with IRS observations, but no MIPS observations in which the IRS band is close to rest-frame $5 \mu \mathrm{m}$. These IRS-derived luminosities are calculated directly from the $16 \mu \mathrm{m}$ flux densities and thus do not account for the shape of the SED or the related offset between rest-frame $5 \mu \mathrm{m}$ and the restframe wavelength probed by the $16 \mu \mathrm{m}$ observations. The uncertainty on the IRS-derived rest-frame $5 \mu \mathrm{m}$ luminosities is estimated to be $\lesssim 50 \%$.

The high luminosities, $L_{5 \mu \mathrm{m}} \gtrsim 10^{11} L_{\odot}$, in Figure 10 imply that the HzRGs are reradiating a substantial amount of energy, most likely reprocessed emission from the obscured AGN heating the surrounding dust. The implied total $(5-1000 \mu \mathrm{m}) \mathrm{IR}$ 

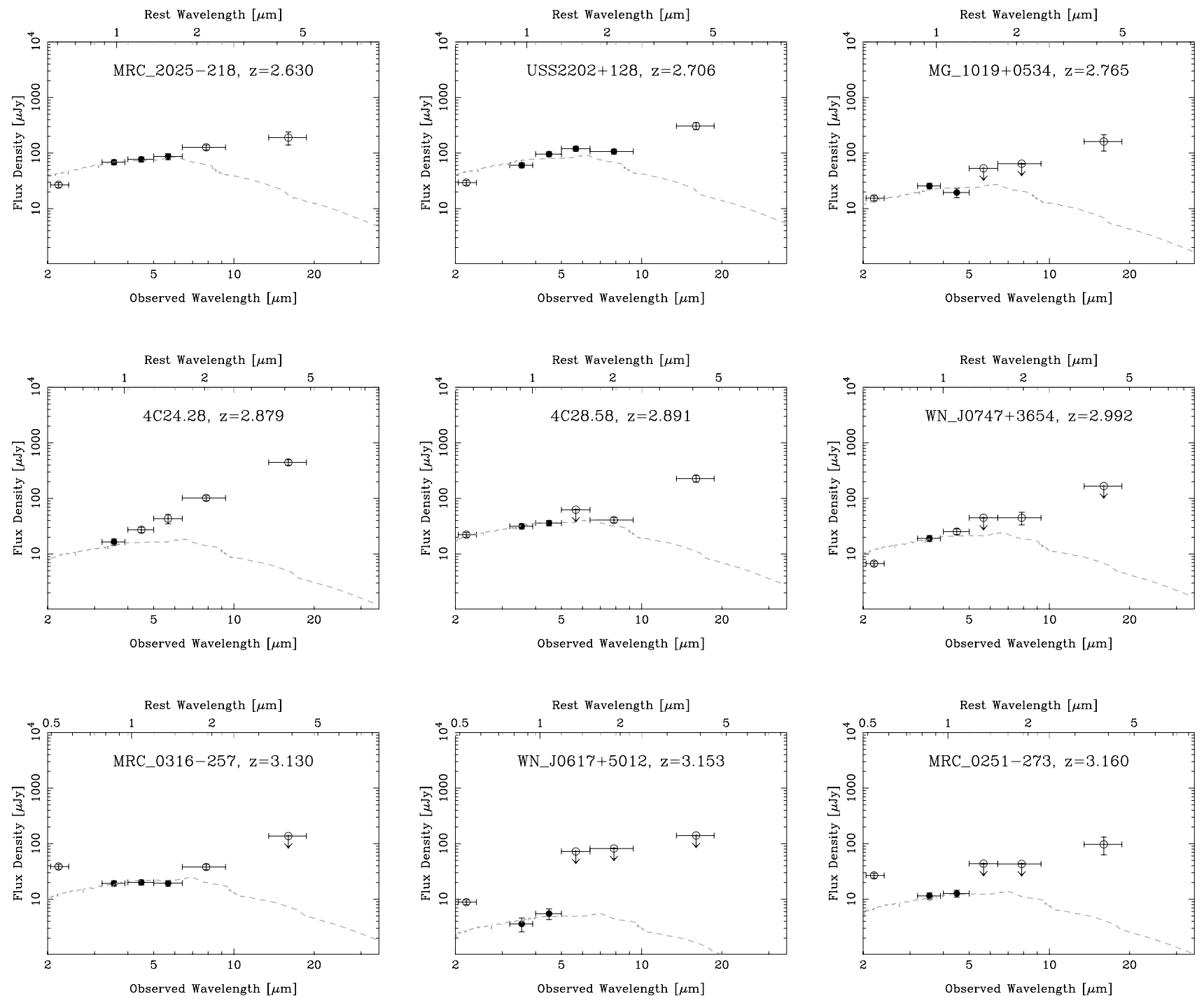

Fig. 6-Continued

luminosities will be greater than the mid-IR luminosities presented here and hence most of these HzRGs would be classified as LIRGs $\left(L_{5-1000 \mu \mathrm{m}}>10^{11} L_{\odot}\right)$, or in many cases as ULIRGs $\left(L_{5-1000 \mu \mathrm{m}}>10^{12} L_{\odot}\right)$ had they been initially identified with no knowledge of their radio properties. These luminosities cover a similar range as those found by Ogle et al. (2006) for a sample of quasars and radio galaxies from the 3CRR catalog: $\nu L_{\nu}($ rest $15 \mu \mathrm{m})=$ $10^{44}-10^{46} \mathrm{ergs}^{-1}$. Luminosities this high confirm that powerful radio galaxies harbor "hidden quasars," i.e., supermassive black holes accreting near the Eddington limit. A few sources have lower luminosities, classified by Ogle et al. (2006) as being in the mid-IR "weak" regime, $\nu L_{\nu}($ rest $15 \mu \mathrm{m})<10^{44} \mathrm{ergs} \mathrm{s}^{-1}$, approximately equivalent to $\nu L_{\nu}($ rest $5 \mu \mathrm{m})<10^{11} L_{\odot}$, indicating that they probably have lower accretion rates and are possibly nonthermal, jet-dominated sources.

The alternate explanation for these high mid-IR luminosities, star formation, is unlikely. Despite the high inferred star formation rates from the submillimeter observations (Archibald et al. 2001; Reuland et al. 2003), $\geq 500 M_{\odot} \mathrm{yr}^{-1}$ for the $\sim 30 \%$ detected and $\leq 500 M_{\odot} \mathrm{yr}^{-1}$ for the nondetections, the star formation is widely scattered as seen in the rest-frame UV/optical images, which show widely distributed morphologies (McCarthy et al. 1987; Best et al. 1996; Zirm et al. 2003; Miley et al. 2006). Hence, the star formation is unlikely to be dense enough to reach temperatures where it would dominate at rest frame $5 \mu \mathrm{m}$.

\subsection{Near- to Mid-IR Luminosity Ratio}

Figure 11 presents the stellar near-IR to total mid-IR $\left(L_{\mathrm{H}}^{\text {stel }} / L_{5 \mu \mathrm{m}}^{\text {tot }}\right)$ rest-frame luminosity ratio plotted against both redshift and $3 \mathrm{GHz}$ luminosity. The ratios range from $\sim 0.1$ to $\sim 6$, and we find that the near- to mid-IR luminosity ratio gradually decreases with both increasing redshift and with increasing radio luminosity. If, as discussed earlier, the mid-IR luminosities are indicators of the power of the AGN (i.e., the AGN accretion rate at the epoch of observation), then for a given host stellar mass, the accretion rate apparently declines at lower redshift and lower radio power. This correlation suggests evolution with redshift, but it may also be due to selection: our mid-IR luminosities are based on the $24 \mu \mathrm{m}$ detections, which are fewer at higher redshift. The trend with radio power is perhaps more interesting, showing that HzRGs with more luminous radio emission are also accreting at a higher rates (for a given stellar mass). This result is also consistent with the radio luminosity-emission line luminosity correlation (Willott et al. 1999). This trend with redshift is probably also related to the slight trend, albeit with a large scatter, when directly comparing the mid-IR to radio luminosity (see Fig. 12), 

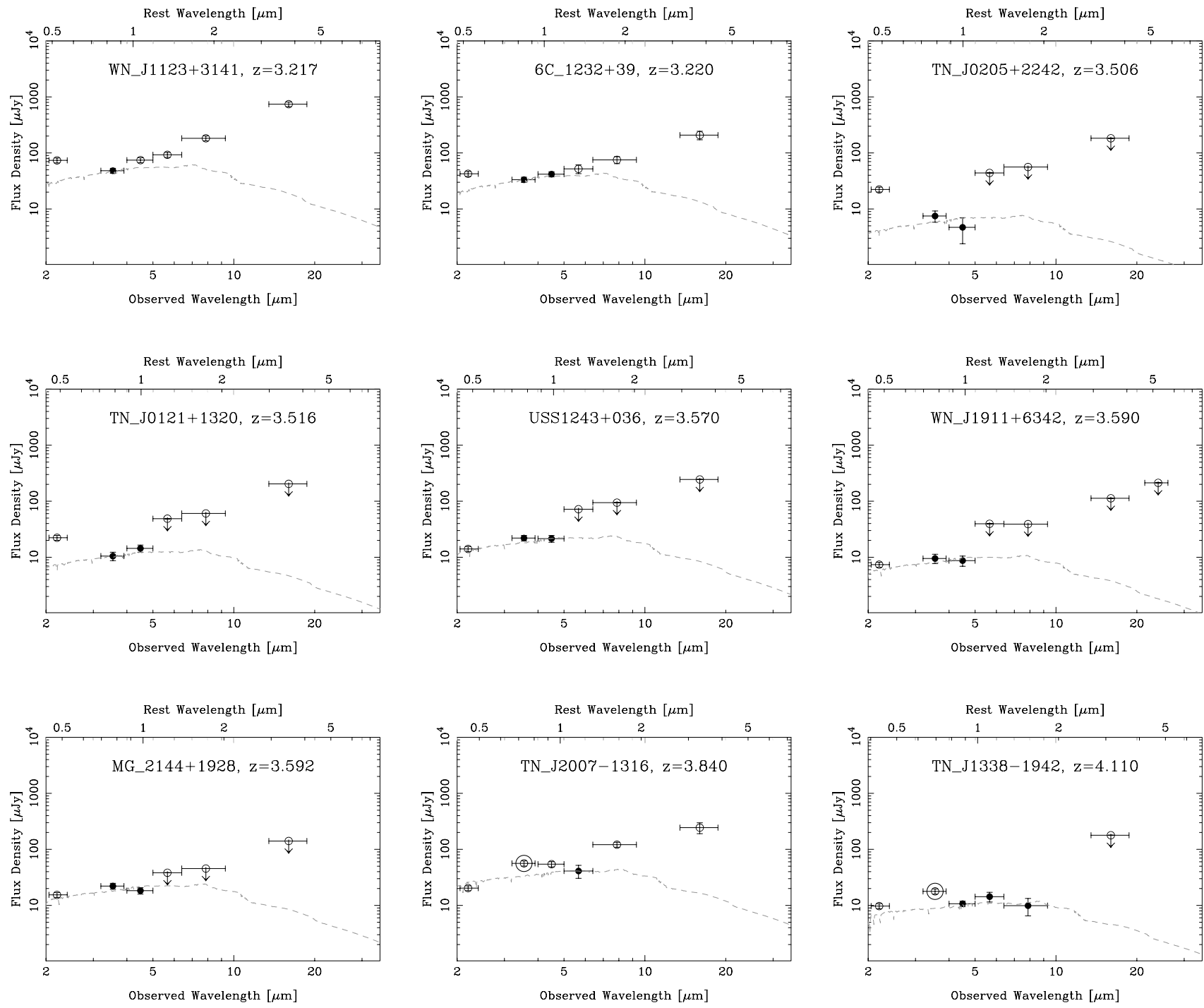

FIG. 6-Continued

although that trend is possibly due to the scaling of luminosities with distance.

\section{PHOTOMETRIC STELLAR MASSES}

The stellar luminosities shown in Figure 9 are converted to masses using the mass-to-light ratios ${ }^{18}$ derived from the PÉGASE. 2 early-type galaxy model with $z_{\text {form }}=10$ described in $\S 4.1$ and are presented in Figure 13. The plotted errors are from the SED fitting of the luminosity; systematic errors to the stellar masses are discussed in $\S 6.3$. Hence, we show that the stellar masses of the hosts of radio galaxies generally have masses distributed between $10^{11}$ and $10^{11.5} M_{\odot}$ with remarkable little scatter across the redshift range of $1 \leq z \leq 4$. The one HzRG with larger uncertainty at $z=5.2$ suggests that this trend may hold to higher redshifts still with the implication that this source had to have formed early and quickly. Given that the lifetimes of radio galaxies are short, 10-100 Myr, these sources are likely to be rep-

18 The modeled mass-to-light ratios include the mass of all material that has gone through star formation. It thus represents the integrated star formation history and includes stars, as well as substellar objects and compact poststellar relics such as white dwarfs, neutron stars, and black holes, but not the gas that is recycled to the ISM. Stars dominate the rest-frame near-IR SED. resentative of a larger population of massive galaxies at these redshifts. Massive galaxies are now being found in great numbers across $1 \leq z \leq 3$ (e.g., Labbé et al. 2005; Papovich et al. 2006; van Dokkum et al. 2006).

We compare our HzRG sample to SMGs studied by Borys et al. (2005) (Fig. 13, red crosses). HzRGs and SMGs are among the most massive objects over this redshift range, showing that both classes of galaxy probe the most massive galaxies in the highredshift universe, although we note that HzRGs are much rarer beasts. Borys et al. (2005) studies 13 SMGs identified in the GOODS survey. For comparison, the most luminous radio source known in the GOODS survey is VLA J123642+621331, a $470 \mu \mathrm{Jy}$ source at $z=4.24$ identified by (Waddington et al. 1999). With $L_{3 \mathrm{GHz}} \sim 10^{25} \mathrm{~W} \mathrm{~Hz}^{-1}$, the radio emission from this galaxy is most likely coming from an AGN, not star formation, as suggested by European VLBI Network observations (Garrett et al. 2001), which show the source to be unresolved. Nevertheless, its radio power does not qualify it as a HzRG according to our selection criteria defined in $\S 2$.

\subsection{Radio Power Dependence on Stellar Mass}

Figure 14 presents the derived stellar masses plotted against rest-frame $3 \mathrm{GHz}$ radio luminosity. The small dynamic range of 

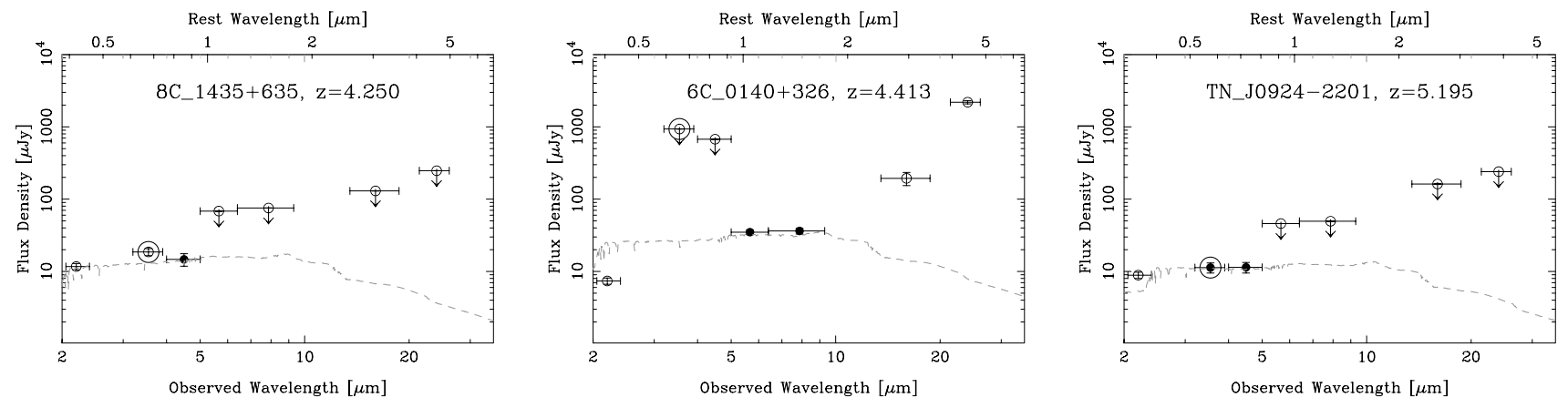

FIG. 6-Continued

the stellar masses combined with their relatively large uncertainties makes the formal determination of any correlation difficult, but a weak correlation is visually apparent. A proper statistical survival analysis that accounts for the multiple upper limits finds that the Cox hazard probability is only 0.5 , meaning there is only a $50 \%$ chance that stellar mass correlates with radio luminosity for our sample. The detection of a correlation, showing that more powerful radio sources are hosted by more massive galaxies, would not be surprising, and is in fact expected if the $M-\sigma$ relation holds to high redshift and all HzRGS have similar efficiency rates or Eddington ratios. Previous work, inferring stellar masses from the observed near-IR flux rather than the rest-frame near-IR luminosity, has suggested this correlation in the past (Eales et al. 1997; Best et al. 1998; Jarvis et al. 2001; De Breuck et al. 2002; Willott et al. 2003).

\subsection{Comparisons with More Detailed Modeling}

As mentioned earlier, several HzRGs from our sample have already been the subject of detailed studies that included Spitzer observations and determined host stellar masses. These studies have generally used more extensive photometry for the modeling, including optical and near-IR data, and have used more complicated models to determine the host stellar masses. We review the results of more detailed modeling here; in brief, allowing for different choices of IMF, the simplistic modeling presented here is consistent with the more detailed modeling.

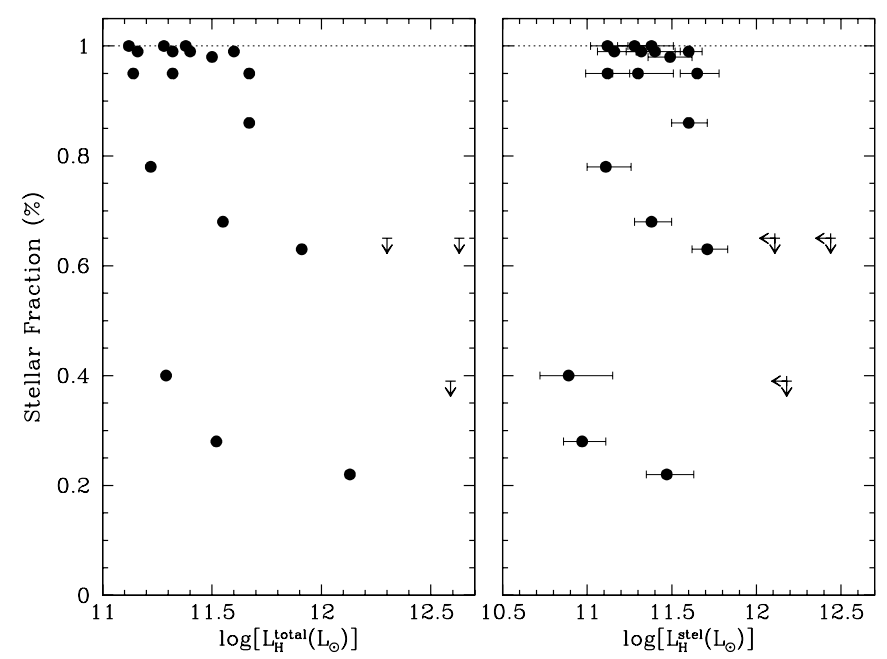

FIG. 7.-Fraction of the HzRG rest-frame $H$-band luminosity that is modeled as stellar, plotted as a function of total $H$-band luminosity and stellar $H$-band luminosity. The stellar fraction appears independent of the stellar near-IR luminosity but is inversely related to the total near-IR luminosity. This implies that the underlying hosts constitute a homogeneous, similar stellar mass population, but with varying AGN contributions to their observed total near-IR luminosities.
MRC 2104-242 $(z=2.491)$ was analyzed in detail by VillarMartín et al. (2006), who found a stellar mass of $(5 \pm 2) \times$ $10^{11} M_{\odot}, E(B-V)=0.4$, and an age of $1.8 \mathrm{Gyr}$. This result is consistent with the work here where we find a mass of $1.6_{-0.8}^{+1.6} \times$ $10^{11} M_{\odot}$. Our work assumes no extinction, and $z_{\text {form }}=10$ corresponds to an age of 2.1 Gyr for the redshift of this HzRG. Much of the difference can be attributed to the Salpeter (1955) IMF adopted by Villar-Martín et al. (2006), which yields a mass-tolight ratio systematically higher by a factor of $\sim 40 \%$ compared to the Kroupa (2001) IMF adopted here. Taking this choice into account, the derived masses are within $1 \sigma$ of each other, which is rather encouraging given the increased sophistication of the stellar modeling in Villar-Martín et al. (2006), as well as their inclusion of near-IR data from the ground and HST.

LBDS 53W091 ( $z=1.552)$ was studied by Stern et al. (2006) and, as discussed earlier, is (proto-) typical of the old, evolved class of extremely red objects. Using the Salpeter (1955) IMF, Stern et al. (2006) find a stellar mass of $\sim 3 \times 10^{11} M_{\odot}$ and an age of 3.5-4 Gyr. Accounting for the difference in IMF, this result is consistent with the masses presented here, $\sim 1.6 \times 10^{11} M_{\odot}$. The ages of the LBDS galaxies imply a redshift of formation $z_{\text {form }} \sim 9$, similar to our $z_{\text {form }}=10$.

The full radio to X-ray SED of 4C $23.56(z=2.483)$ is presented in C. De Breuck et al. (2007, in preparation), the first time such data has been presented for a radio galaxy above $z=2$. A full SED decomposition of the AGN and stellar emission (such as scatter quasar light, emission lines and nebular continuum) is performed. With a Salpeter IMF and no extinction, they derive a
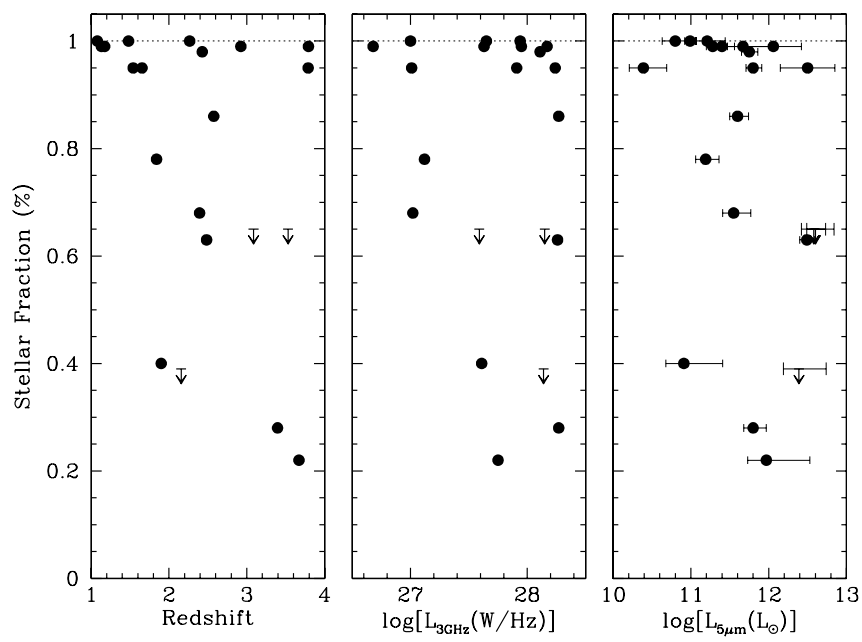

FIG. 8.- Fraction of the HzRG rest-frame $H$-band luminosity that is modeled as stellar, plotted as a function of (1) redshift, (2) rest-frame $3 \mathrm{GHz}$ radio power, and (3) rest-frame $5 \mu \mathrm{m}$ luminosity. Only the $21 \mathrm{HzRG}$ with MIPS detections are plotted. No correlations are evident. 


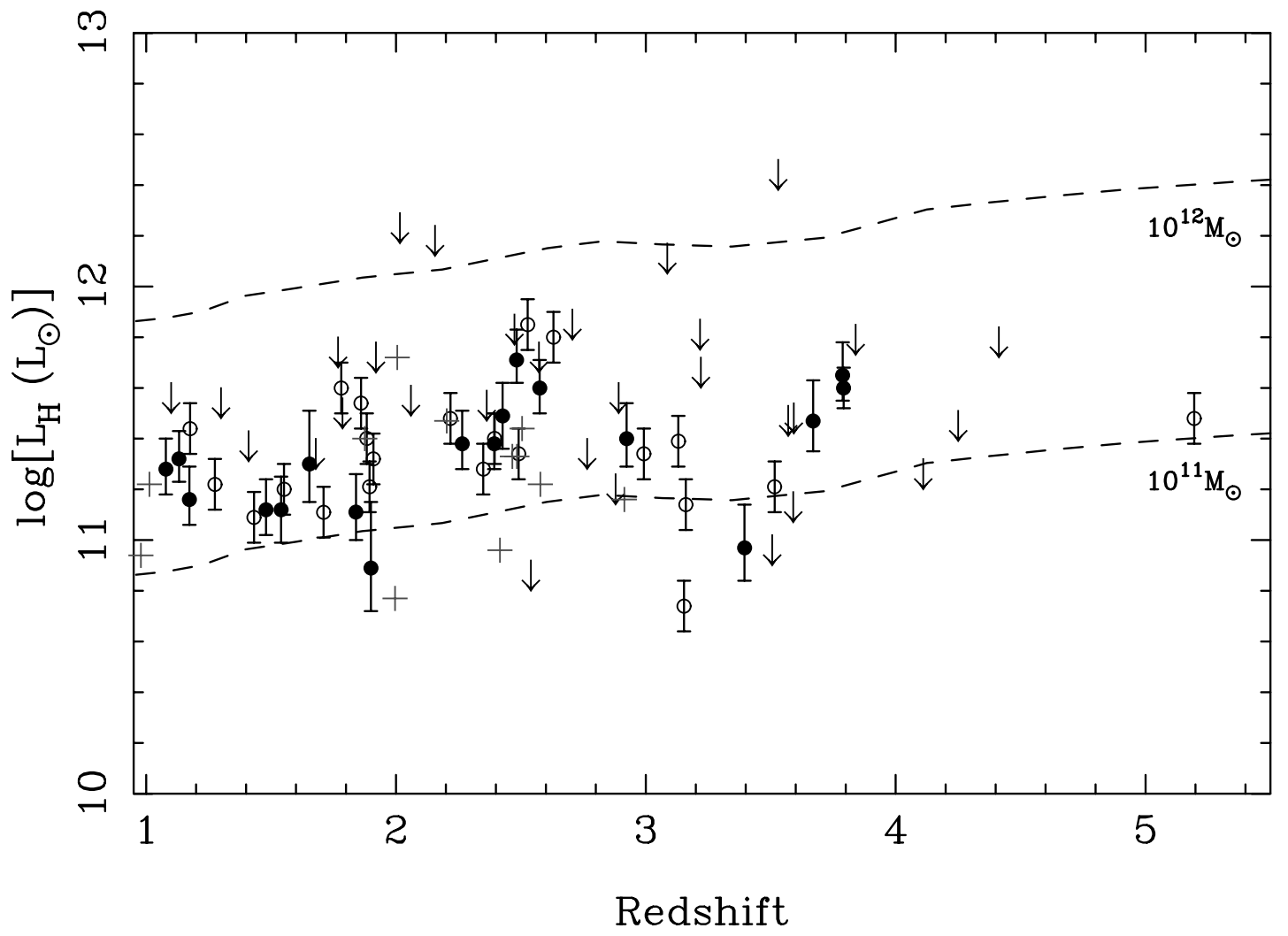

Fig. 9.- Rest-frame $H$-band stellar luminosity vs. redshift for the Spitzer HzRG sample, derived from the best-fit models to the multiband photometry. Solid circles indicate those luminosities derived from HzRGs with MIPS detections, while open circles are luminosities derived from HzRGs without MIPS detections. Those radio galaxies where only a maximum fit of the stellar SED was possible have upper limits, indicated by arrows. The dashed lines represent the luminosities of elliptical galaxies with $z_{\text {form }}=10$ taken from the PÉGASE. 2 models and normalized to $10^{11}$ and $10^{12} M_{\odot}$. Crosses mark the stellar luminosity of submillimeter galaxies from Borys et al. (2005), rederived in the same fashion as our HzRG sample. [See the electronic edition of the Supplement for a color version of this figure.]

stellar mass of $5.1_{-2.1}^{+1.6} \times 10^{11} M_{\odot}$. Again, allowing for the different choice of IMF, this result is consistent within $1 \sigma$ of the results here, $3.9_{-0.7}^{+1.2} \times 10^{11} M_{\odot}$.

These comparisons give us confidence that although the SED modeling we adopt is somewhat simplistic, the derived stellar masses appear to be accurate within the uncertainties inherent to the enterprise.

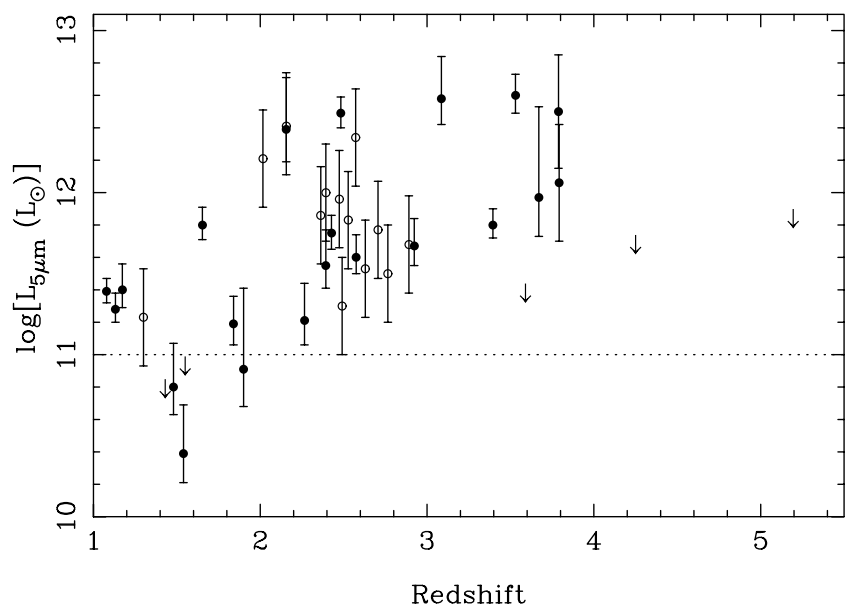

FIG. 10.-Rest $5 \mu \mathrm{m}$ monochromatic luminosity vs. redshift. Filled symbols indicate sources observed with MIPS; open symbols indicate sources without MIPS observations but observed with IRS. Dotted line represents the divide between midIR "strong" and mid-IR "weak" AGNs, as defined by Ogle et al. (2006).

\subsection{Systematic Effects on the Stellar Masses}

Deriving stellar masses of high-redshift galaxies $(z \gtrsim 1)$ has become widespread in recent years with the advent of deep nearand mid-IR extragalactic surveys (e.g., Shapley et al. 2003; Labbé et al. 2005; Rettura et al. 2006; L. A. Moustakas et al. 2007, in preparation). Photometric masses, as derived here, are subject to several systematic uncertainties. However, "relative" masses can be derived with less uncertainty (e.g., Dickinson et al. 2003). We now briefly discuss some of the systematic effects inherent to the stellar masses derived here. This analysis will be particularly important for future comparison of our results with other samples.

\subsubsection{IMF}

The IMF remains one of the largest uncertainties when modeling the SED of a galaxy. There have been several updates to the classical Salpeter (1955) IMF recently (e.g., Kroupa 2001; Chabrier 2003; etc.). Even these, however, are largely based on local stars within our own Galaxy and hence suffer uncertainties at the lowmass end and for extreme metalicities (high and low). We have chosen to use the Kroupa (2001) IMF here. However, our results would not be very different $(<2 \%)$ if we used the Chabrier (2003) IMF. Had we used the classical Salpeter (1955) IMF, our stellar masses would increase systematically by $40 \%$, independent of our choice of $z_{\text {form }}$.

\subsubsection{Formation Redshift}

We have assumed a uniform formation redshift, $z_{\text {form }}=10$, in the current analysis. Eyles et al. (2006) find a range of $z_{\text {form }}=$ $7-14$ for $i^{\prime}$-band dropouts at $z \sim 6$ in the GOODS fields for 

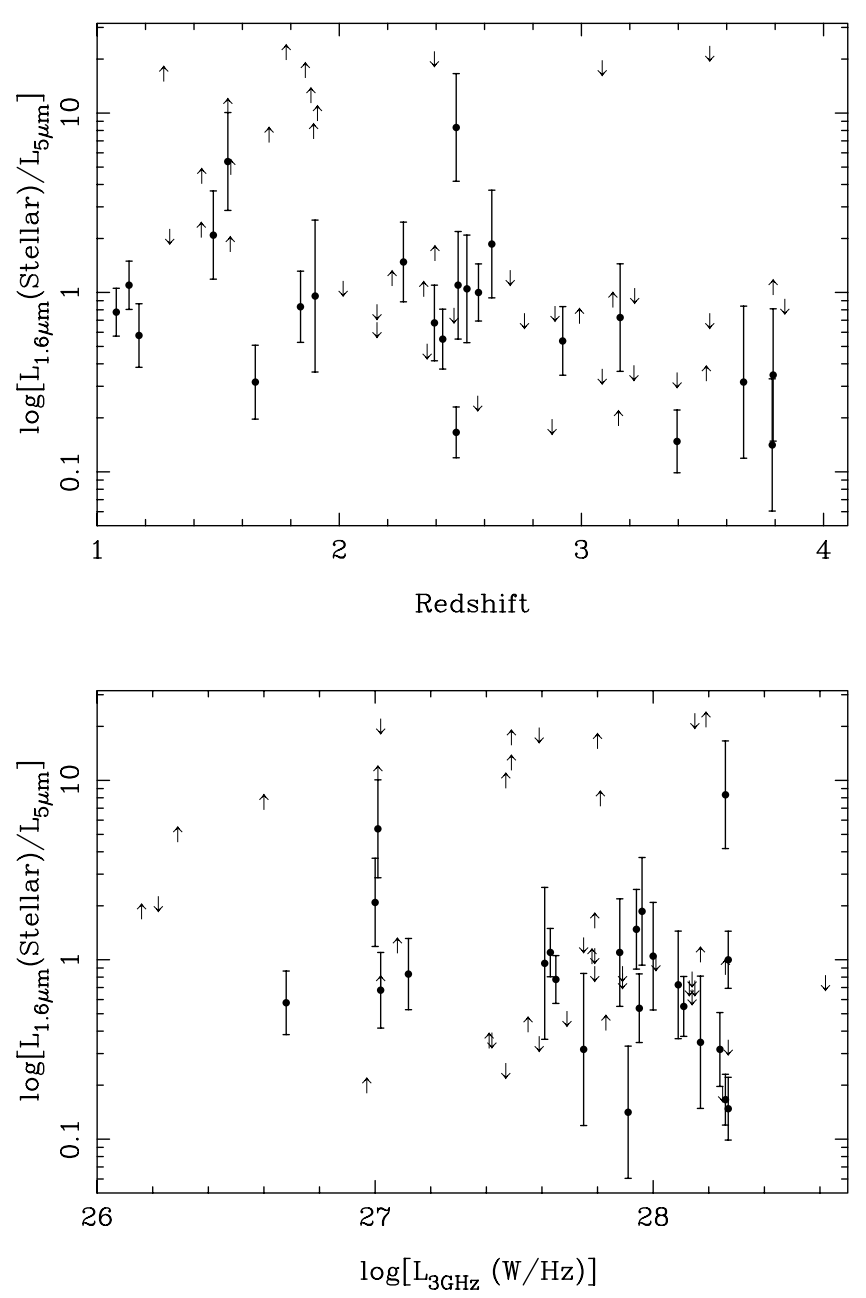

FIG. 11.- Ratio of rest-frame stellar near-IR ( $H$-band) luminosity to total midIR $(5 \mu \mathrm{m})$ luminosity against redshift (top) and $3 \mathrm{GHz}$ luminosity (bottom). This ratio appears to strongly correlate with both redshift and radio luminosity, although both correlations are probably due to the loose correlation of the $5 \mu \mathrm{m} \mathrm{lu}$ minosity with both redshift and radio luminosity.

galaxies. These distant galaxies are our best-studied examples of galaxies at $6 \lesssim z \lesssim 7$ and hence are our best (only) candidates for the parent populations to HzRGs. This range of formation redshifts corresponds to 0.16 Gyr earlier or 0.3 Gyr later than $z=10$ when the universe was only $\sim 1$ Gyr old. Assuming the full range of $z_{\text {form }}$ from Eyles et al. (2006) changes the mass by $\pm 17 \%$ for a Kroupa (2001) IMF and $\pm 8 \%$ for a Salpeter (1955) IMF. The change is such that the stellar mass decreases with a later formation redshift and vice versa.

\subsubsection{Extinction}

No internal galactic extinction is considered here. However, $\sim 50 \%$ of the HzRGs have submillimeter detections, implying substantial dust content, although the dust distribution need not heavily obscure the old stellar population observed at rest-frame near-IR wavelengths. To obtain a $20 \%$ underestimation of the stellar mass from the rest-frame $H$-band luminosity requires an $A_{V}>1.17$ assuming a Calzetti et al. (1994) extinction curve.

\subsubsection{Starburst Component}

These stellar mass estimates do not include the possible contribution of young stellar populations, which would lead to an over estimation of the stellar mass. If we include a $10 \%$ by mass starburst component, starting 200 Myr before the observed red-

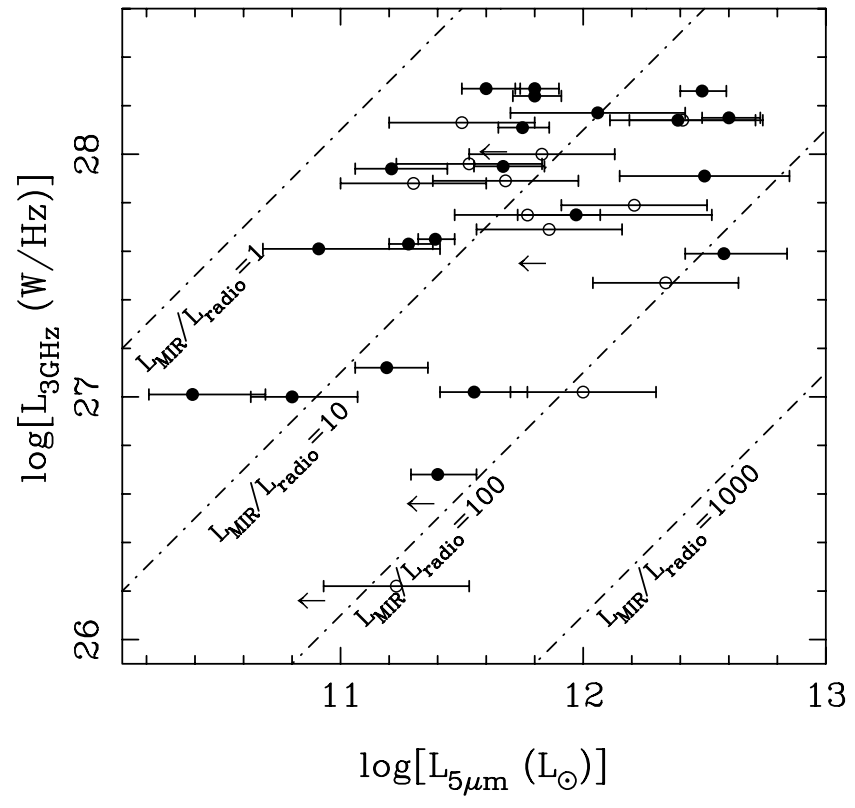

FIG. 12.-Rest $5 \mu \mathrm{m}$ monochromatic luminosities vs. rest $3 \mathrm{GHz}$ radio luminosities showing a slight correlation potentially due distance effects, or more likely demonstrating the both mid-IR and radio luminosity trace AGN power and that the scatter is induced by the time delay of jets powering the lobes after a change in AGN power.

shift, we find that, while the SED at rest-frame near-IR does not change significantly, the mass-to-light ratio does decrease by $10 \%-20 \%$ at $H$ band (see Fig. 2). Redoing our analysis again with this younger component results in nearly identical $H$-band luminosities as before, but a $\lesssim 20 \%$ decrease in stellar mass. We note that despite several sources in our sample having submillimeter detections, it is not thought that on-going star formation constitutes as much as $10 \%$ by mass for such systems.

Deep rest-frame UV spectropolarimetry of nine optically bright star-forming HzRGs by Vernet et al. (2001) finds only one object with a substantial contribution from a population of young stars. Hence, we conclude that there may be a mild systematic overestimation of the stellar mass, but this uncertainty is likely less significant than other uncertainties discussed above, such as the choice of the IMF in the modeling.

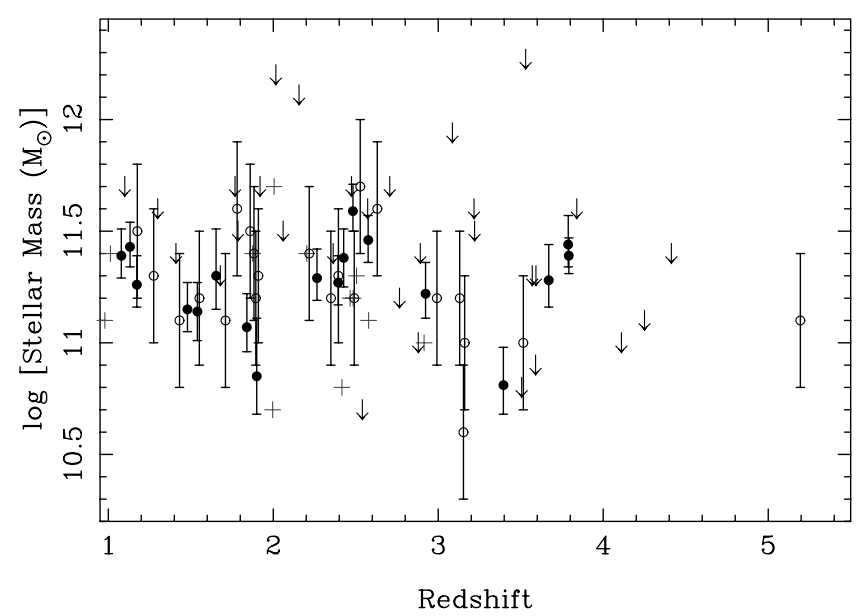

FIG. 13.- Stellar mass in solar units plotted against redshift for the 69 Spitzer HzRGs. Symbols are as in Fig. 9. [See the electronic edition of the Supplement for a color version of this figure.] 


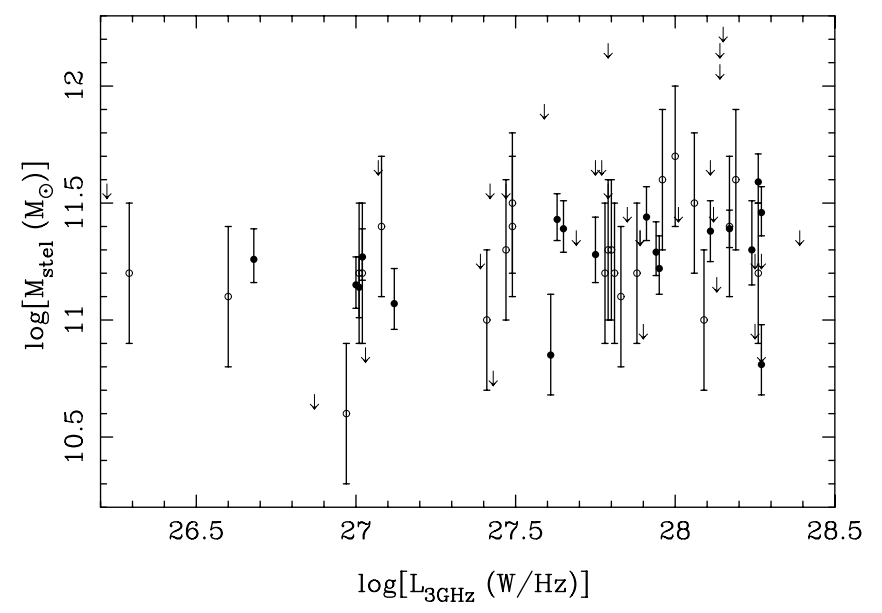

FIG. 14.- Stellar mass against rest-frame $3 \mathrm{GHz}$ luminosity. There is evidence of a slight trend, such that the most massive galaxies host more powerful radio sources, however, accounting for the large number of upper limits in the plot, a formal survival analysis shows only a $50 \%$ chance of the two observables being correlated. Deeper data and better coverage with the longer wavelength Spitzer cameras would show conclusively whether or not this correlation exists.

\subsection{5. $T P-A G B$ Stars}

Maraston (2005) has argued that the thermally pulsing asymptotic giant branch (TP-AGB) phase in the evolution of a simple stellar population is not properly considered in other spectral evolution synthesis codes (e.g., PÉGASE and Bruzual \& Charlot 2003), and hence analysis using these models overpredicts the near-IR mass-to-light ratio for galaxy ages $\lesssim 1$ Gyr. For our choice of $z_{\text {form }}$, this age corresponds to galaxies at $3 \lesssim z \lesssim 4$. We emphasize that the PÉGASE models $d o$ incorporate the TP-AGB phase, just in a different manner than the Maraston (2005) models. If this TP-AGB phase is indeed an issue, it is most likely to only affect the highest redshift (and hence youngest) sources in our sample by decreasing their stellar mass by $\leq 30 \%$. As we have seen in $\S 6.3 .4$, an unaccounted starburst leads to an overestimation of the stellar mass. Use of the model from Maraston (2005) would hence increase this overestimate to about $30 \%$, which is on par with the uncertainty due to all the other systematics.

\section{DISCUSSION AND CONCLUSIONS}

We have performed a comprehensive infrared survey of $69 \mathrm{HzRG}$ above $z=1$, the first time such galaxies have been detected in the rest-frame near-IR and mid-IR. This work presents the largest sample of confirmed type 2 quasars to be systematically studied with Spitzer. As expected, they predominantly reside in the IRAC color-color space dominated by type 1 AGNs found empirically by Lacy et al. (2004) and Stern et al. (2005) and theoretically by Sajina et al. (2005).

We model the rest-frame near to mid-IR SEDs derived from our broad-band IRAC, IRS, and MIPS imaging. We use a toy model comprised of thermal BBs to represent the emission at longer wavelengths presumably due to dust, and the PEGASE code to model the stellar component. Our analysis yields total near-IR ( $H$ band) and mid-IR $(5 \mu \mathrm{m})$ rest-frame luminosities, and we deconvolve the stellar and AGN contributions at $H$ band. The scatter of stellar $H$-band luminosities is lower than that for the total $H$-band luminosities, and we find the HzRGs with lower stellar fractions tend to have higher rest-frame $H$-band total luminosities, although this trend is weak. When $H$-band luminosities are corrected for AGN contribution, they show no correlation with stel- lar fraction. In addition, the stellar fraction is not found to correlate with redshift, radio luminosity, or mid-IR luminosity. These results are consistent with orientation-driven models of AGN unification.

We find that the $H$-band stellar luminosities are consistent with stellar masses of $10^{11}-10^{12} M_{\odot}$, comparable with the masses of local massive radio galaxies (McCarthy 1993; Jarvis et al. 2001; Willott et al. 2003). This conversion of luminosity to mass assumes a dust-free, passively evolving elliptical formed at $z=10$ with no recent episodes of star-formation. We discuss these effects and other systematics and conclude that our findings are robust at the level of $\approx 10 \%-20 \%$. We also note that effects can act to either increase the inferred mass (the presence of dust) or to decrease it (recent star-burst activity). So multiple effects may cancel out, thus most likely simply increasing the scatter in the estimated stellar masses.

The derived stellar masses average $10^{11.4} M_{\odot}$ across $z=1-4$ with a scatter less than $50 \%$. This scatter is remarkably small, suggesting that the tightness of the observed $K-z$ relation at $z>1$ is due to the intrinsic homogeneity of the HzRG population. The increase in the scatter from $z<1$ to $z>1$ can largely be attributed to the $K$-band being affected by emission lines, scattered light from the AGN (e.g., C. De Breuck et al. 2007, in preparation), and, potentially, young stellar populations. The scatter in the observed $H$-band luminosities can be explained by varying contributions from AGN-heated dust entering the IRAC bands.

These masses, while very high, are not uncommon at these redshifts. Much work has been done recently to detect massive, $M>10^{11} M_{\odot}$, galaxies at high redshifts (e.g., Labbé et al. 2005; Papovich et al. 2006; van Dokkum et al. 2006). Densities of approximately one $M>10^{11} M_{\odot}$ galaxy per square arcmin are found in the redshift range $2 \lesssim z \lesssim 3$ (Papovich et al. 2006; van Dokkum et al. 2006). Our survey targeted a sample of known sources from the literature, derived from multiple radio surveys. As such, we are ill posed to address space densities from the current work. However, we note that our adopted definition of a HzRG $\left(z>1\right.$ and $\left.L_{3 \mathrm{GHz}}>10^{26} \mathrm{~W} \mathrm{~Hz}^{-1}\right)$ implies that large area surveys such as the Faint Images of the Radio Sky at Twentycentimeters (FIRST; Becker et al. 1995) have already detected all HzRGs at $1<z \lesssim 3$, although the vast majority lack spectroscopic observations thus far. We can therefore constrain the surface density of HzRGs to be less than $<100 \mathrm{deg}^{-2}$, which is significantly lower than the surface density of optically and near-IR selected massive galaxies. This low surface density is most likely due to the short lifetimes (10-100 Myr) and low duty cycles of the most powerful radio sources, even if the every massive galaxy were to go through a radio-luminous phase.

We find a slight trend that the galaxies with the higher stellar masses host the more powerful radio sources, a trend suggested by other authors (McCarthy 1993; Jarvis et al. 2001; Willott et al. 2003). However, a full survival analysis taking proper account of upper limits finds this correlation has only a $50 \%$ chance of being correct. The correlation is expected if the $M-\sigma$ relation holds to high redshift and HzRGs all accrete at comparable Eddington ratios.

Most of the HzRG mid-IR luminosities are above $10^{11} L_{\odot}$, implying even higher total IR luminosities. These LIRG and ULIRGlike luminosities are due to reprocessed radiation from gas and dust obscuring a highly accreting AGN. Rest-frame mid-IR luminosity $\left(\lambda_{0} \sim 5 \mu \mathrm{m}\right)$ may be a good, independent indicator of AGN activity. Comparison with traditional AGN indicators at other wavelengths is currently underway (X-ray: D. Alexander et al. 2007, in preparation; radio: N. Seymour et al. 2007, in preparation). 
We would like to thank the referee for the constructive comments provided on the clarification of this paper. N. S. and D. S. thank ESO for generous hospitality on several occasions over the last few years. We thank Dave Frayer and Ranga-Ram Chary for advice on reducing the MIPS data and the IRAC instrument team for allowing publication of GTO data on 4C 41.17 and 6C $0140+326$.

This work is based on observations made with the Spitzer Space Telescope, which is operated by the Jet Propulsion Laboratory, California Institute of Technology under a contract with
NASA. Support for this work was provided by NASA through an award issued by JPL/Caltech. The work by W. v. B. was performed under the auspices of the US Department of Energy, National Nuclear Security Administration by the University of California, Lawrence Livermore National Laboratory under contract W-7405-Eng-48. W. v. B. also acknowledges support for radio galaxy studies at UC Merced, including the work reported here, with the Spitzer Space Telescope via NASA grants SST $1264353,1265551,1279182$, and 1281587.

\section{APPENDIX}

\section{NOTES ON INDIVIDUAL SOURCES}

Here we provide individual comments on selected HzRGs. The SED fitting is presented in Figure 5 (20 sources with MIPS detections) and Figure 6 (50 sources without MIPS detection), arranged in order of increasing redshift.

$3 C 65(z=1.176)$. - This HzRG was observed in only two bands due to an astrometric error in NED, which we have since had corrected.

$3 C 294(z=1.786)$. - While this radio galaxy is detected in all IRAC bands, the photometry is severely affected by a bright star $8^{\prime \prime}$ a to the east by northeast. This bright star has allowed detailed adaptive optics imaging of this object (e.g., Quirrenbach et al. 2001; Steinbring et al. 2002).

B3 J2330+3927 $(z=3.086)$. - This HzRG is one of two sources consistent with an AGN power law dominating the entire optical to mid-IR emission. We therefore can only provide an upper limit to the stellar mass. Although optical and near-IR spectroscopy (De Breuck et al. 2003) show a classical type 2 AGN spectrum, Pérez-Torres \& De Breuck (2005) found that the radio morphology is more consistent with a type $1 \mathrm{AGN}$. The latter would be in agreement with the high contribution from hot AGN light seen in the near- to mid-IR SED.

MRC 0316-257 $(z=3.131)$. - Due to an error at the time of proposal submission, the source observed with IRAC was MRC 0316-257B (Le Fèvre et al. 1996), a spectroscopically confirmed $z=3.118$ AGN, 71" from MRC 0316-257. We used the deeper Cycle 1 GO data (Program ID 3482, PI van Breugel), but no IRS $16 \mu \mathrm{m}$ data are available for the $z>2$ radio galaxy.

$T X J 1908+7220(z=3.53)$. - This HzRG is the second of two sources consistent with an AGN power law dominating the entire optical to mid-IR emission. We therefore can only provide an upper limit to the stellar mass.

TN J1338-1942 $(z=4.11)$. - This HzRG is the fourth highest redshift source in our sample (De Breuck et al. 1999). Although the $3.6 \mu \mathrm{m}$ IRAC band is likely to be contaminated by $\mathrm{H} \alpha$, the remaining bands are consistent with a stellar SED. The low $8.0 \mu \mathrm{m}$ flux ensures that any hot dust contamination will be small.

$8 C 1435+635(z=4.25)$. - This HzRG is the third highest redshift source in our sample (Lacy et al. 1994). The stellar mass estimate is based on the $4.5 \mu \mathrm{m}$ IRAC detection only, as the $3.6 \mu \mathrm{m}$ band is likely to be contaminated by $\mathrm{H} \alpha$ emission.

$6 C 0140+326(z=4.41)$. - This HzRG is the second most distant galaxy and is masked by a bright, foreground galaxy in IRAC channels 1 and 2, thus rendering source extraction at these wavelengths infeasible.

TN J0924-2201 ( $z=5.19)$. - This HzRG is the most distant radio galaxy in our sample (van Breugel et al. 1999). It is detected only at 3.6 and $4.5 \mu \mathrm{m}$. Deeper IRAC data have been obtained (Program ID 20749; PI: W. Zheng), but these data were not public yet at the time of writing (2006 December).

Antonucci, R. 1993, ARA\&A, 31, 473

Archibald, E. N., Dunlop, J. S., Hughes, D. H., Rawlings, S., Eales, S. A., \& Ivison, R. J. 2001, MNRAS, 323, 417

Athreya, R. M., Kapahi, V. K., McCarthy, P. J., \& van Breugel, W. 1997, MNRAS, 289, 525

Barthel, P. D. 1989, ApJ, 336, 606

Becker, R. H., White, R. L., \& Helfand, D. J. 1995, ApJ, 450, 559

Bennett, A. S. 1962, MmRAS, 68, 163

Bennett, C. L., Lawrence, C. R., Burke, B. F., Hewitt, J. N., \& Mahoney, J. 1986, ApJS, 61, 1

Bertin, E., \& Arnouts, S. 1996, A\&AS, 117, 393

Best, P. N., Longair, M. S., \& Röttgering, H. J. A. 1996, MNRAS, 280, L9 1998, MNRAS, 295, 549

Blandford, R., \& Payne, D. 1982, MNRAS, 199, 883

Blundell, K. M., Rawlings, S., Eales, S. A., Taylor, G. B., \& Bradley, A. D. 1998, MNRAS, 295, 265

Borys, C., Chapman, S., Halpern, M., \& Scott, D. 2003, MNRAS, 344, 385

Borys, C., Smail, I., Chapman, S. C., Blain, A. W., Alexander, D. M., \& Ivison, R. J. 2005, ApJ, 635, 853

Broderick, J. W., De Breuck, C., Hunstead, R. W., \& Seymour, N. 2007, MNRAS, 375, 1059

Brookes, M. H., Best, P. N., Rengelink, R., \& Röttgering, H. J. A. 2006, MNRAS, 366, 1265

Bruzual, G., \& Charlot, S. 2003, MNRAS, 344, 1000

\section{REFERENCES}

Calzetti, D., Kinney, A. L., \& Storchi-Bergmann, T. 1994, ApJ, 429, 582 Chabrier, G. 2003, PASP, 115, 763

Chambers, K. C., Miley, G. K., van Breugel, W. J. M., Bremer, M. A. R., Huang, J.-S., \& Trentham, N. A. 1996, ApJS, 106, 247

Cleary, K., et al. 2007, ApJ, 660, 117

Condon, J. J., Cotton, W. D., Greisen, E. W., Yin, Q. F., Perley, R. A., Perley,

G. B., Taylor, G. B., \& Broderick, J. J. 1998, AJ, 115, 1693

De Breuck, C., van Breugel, W., Minniti, D., Miley, G., Röttgering, H.,

Stanford, S. A., \& Carilli, C. 1999, A\&A, 352, L51

De Breuck, C., et al. 2001, AJ, 121, 1241 2002, AJ, 123, 637

2003, A\&A, 401, 911
Devriendt, J. E. G., Guiderdoni, B., \& Sadat, R. 1999, A\&A, 350, 381

Dey, A., Cimatti, A., van Breugel, W., Antonucci, R., \& Spinrad, H. 1996, ApJ, 465,157

Dey, A., \& Spinrad, H. 1996, ApJ, 459, 133

Dickinson, M., Papovich, C., Ferguson, H. C., \& Budavári, T. 2003, ApJ, 587, 25

Douglas, J. N., Bash, F. N., Bozyan, F. A., Torrence, G. W., \& Wolfe, C. 1996, AJ, 111, 1945

Eales, S., \& Rawlings, S. 1996, ApJ, 460, 68

Eales, S., Rawlings, S., Law-Green, D., Cotter, G., \& Lacy, M. 1997, MNRAS, 291, 593

Eyles, L., et al. 2006, MNRAS, 374, 910 
Fanaroff, B. L., \& Riley, J. M. 1974, MNRAS, 167, 31

Fazio, G. G., et al. 2004, ApJS, 154, 10

Fioc, M., \& Rocca-Volmerange, B. 1997, A\&A, 326, 950

Garrett, M. A., et al. 2001, A\&A, 366, L5

Granato, G. L., Danese, L., \& Franceschini, A. 1997, ApJ, 486, 147

Gregg, M. D., Becker, R. H., White, R. L., Helfand, D. J., McMahon, R. G., \& Hook, I. M. 1996, AJ, 112, 407

Greve, T. R., Ivison, R. J., \& Stevens, J. A. 2006, Astron. Nachr., 327, 208

Haas, M., Siebenmorgen, R., Schulz, B., Krügel, E., \& Chini, R. 2005, A\&A, 442, L39

Heckman, T. M., O’Dea, C. P., Baum, S. A., \& Laurikainen, E. 1994, ApJ, 428, 65

Houck, J. R., et al. 2004, ApJS, 154, 18

Jarvis, M. J., Rawlings, S., Eales, S., Blundell, K. M., Bunker, A. J., Croft, S.,

McLure, R. J., \& Willott, C. J. 2001, MNRAS, 326, 1585

Kroupa, P. 2001, MNRAS, 322, 231

Labbé, I., et al. 2005, ApJ, 624, L81

Lacy, M., Rawlings, S., Hill, G. J., Bunker, A. J., Ridgway, S. E., \& Stern, D. 1999, MNRAS, 308, 1096

Lacy, M., et al. 1994, MNRAS, 271, 504

2004, ApJS, 154, 166

2005, ApJS, 161, 41

2007, AJ, 133, 186

Lawrence, C. R., Readhead, A. C. S., Moffet, A. T., \& Birkinshaw, M. 1986, ApJS, 61, 105

Le Borgne, D., \& Rocca-Volmerange, B. 2002, A\&A, 386, 446

Le Fèvre, O., Deltorn, J. M., Crampton, D., \& Dickinson, M. 1996, ApJ, 471, L11

Lilly, S. J., \& Longair, M. S. 1984, MNRAS, 211, 833

Makovoz, D., \& Khan, I. 2005, in ASP Conf. Ser. 347, Astronomical Data Analysis Software and Systems XIV, ed. P. Shopbell, M. Britton, \& R. Ebert (San Francisco: ASP), 81

Maraston, C. 2005, MNRAS, 362, 799

Martínez-Sansigre, A., Rawlings, S., Lacy, M., Fadda, D., Marleau, F. R., Simpson, C., Willott, C. J., \& Jarvis, M. J. 2005, Nature, 436, 666

Matthews, T. A., Morgan, W. W., \& Schmidt, M. 1964, ApJ, 140, 35

McCarthy, P. J. 1993, ARA\&A, 31, 639

McCarthy, P. J., Baum, S. A., \& Spinrad, H. 1996, ApJS, 106, 281

McCarthy, P. J., van Breugel, W., Spinrad, H., \& Djorgovski, S. 1987, ApJ, 321, L29

Miley, G. K., et al. 2006, ApJ, 650, L29

Miller, L., Peacock, J. A., \& Mead, A. R. G. 1990, MNRAS, 244, 207

Nenkova, M., Ivezić, Ž., \& Elitzur, M. 2002, ApJ, 570, L9

Nesvadba, N. P. H. Lehnert, M. D., Eisenhauer, F., Gilbert, A., Tecza, M., \& Abuter, R. 2006, ApJ, 650, 693

Norman, C., et al. 2002, ApJ, 571, 218

Ogle, P., Whysong, D., \& Antonucci, R. 2006, ApJ, 647, 161

Papovich, C., et al. 2006, ApJ, 640, 92

Pascarelle, S. M., Windhorst, R. A., Keel, W. C., \& Odewahn, S. C. 1996, Nature, 383,45

Pentericci, L., et al. 2001, ApJS, 135, 63

Pérez-Torres, M.-A., \& De Breuck, C. 2005, MNRAS, 363, L41

Pier, E. A., \& Krolik, J. H. 1992, ApJ, 401, 99

Polletta, M. d. C., et al. 2006, ApJ, 642, 673

Quirrenbach, A., Roberts, J. E., Fidkowski, K., de Vries, W., \& van Breugel, W. 2001, ApJ, 556, 108

Rees, M. J. 1978, Nature, 275, 516

Rengelink, R. B., Tang, Y., de Bruyn, A. G., Miley, G. K., Bremer, M. N., Roettgering, H. J. A., \& Bremer, M. A. R. 1997, A\&AS, 124, 259
Rettura, A., et al. 2006, A\&A, 458, 717

Reuland, M., Röttgering, H., van Breugel, W., \& De Breuck, C. 2004, MNRAS, 353, 377

Reuland, M., et al. 2003, ApJ, 592, 755

Richards, G. T., et al. 2006, ApJS, 166, 470

Rieke, G. H., et al. 2004, ApJS, 154, 25

Rocca-Volmerange, B., Le Borgne, D., De Breuck, C., Fioc, M., \& Moy, E. 2004, A\&A, 415, 931

Rocca-Volmerange, B., \& Remazeilles, M. 2005, A\&A, 433, 73

Röttgering, H. J. A., van Ojik, R., Chambers, K., van Breugel, W., \& de Koff, S. 1997, A\&A, 326, 505

Sajina, A., Lacy, M., \& Scott, D. 2005, ApJ, 621, 256

Salpeter, E. E. 1955, ApJ, 121, 161

Saripalli, L., Hunstead, R. W., Subrahmanyan, R., \& Boyce, E. 2005, AJ, 130, 896

Sawicki, M. 2002, AJ, 124, 3050

Shapley, A. E., Steidel, C. C., Pettini, M., \& Adelberger, K. L. 2003, ApJ, 588, 65

Siebenmorgen, R., Freudling, W., Krügel, E., \& Haas, M. 2004, A\&A, 421, 129

Songaila, A., Cowie, L. L., Hu, E. M., \& Gardner, J. P. 1994, ApJS, 94, 461 Spergel, D., et al. 2007, ApJS, 170, 377

Spinrad, H., Djorgovski, S., Marr, J., \& Aguilar, L. 1985, PASP, 97, 932

Steinbring, E., Crampton, D., \& Hutchings, J. B. 2002, ApJ, 569, 611

Stern, D., Chary, R.-R., Eisenhardt, P. R. M., \& Moustakas, L. A. 2006, AJ, 132,1405

Stern, D., Holden, B., Stanford, S. A., \& Spinrad, H. 2003, AJ, 125, 2759

Stern, D., \& Spinrad, H. 1999, PASP, 111, 1475

Stern, D., et al. 2002, ApJ, 568, 71 2005, ApJ, 631, 163

Teplitz, H. I., Charmandaris, V., Chary, R., Colbert, J. W., Armus, L., \& Weedman, D. 2005, ApJ, 634, 128

Treister, E., et al. 2004, ApJ, 616, 123

Tremaine, S., et al. 2002, ApJ, 574, 740

Urry, C. M., \& Padovani, P. 1995, PASP, 107, 803

van Breugel, W., De Breuck, C., Stanford, S. A., Stern, D., Röttgering, H., \& Miley, G. 1999, ApJ, 518, L61

van Breugel, W., Stanford, S. A., Spinrad, H., Stern, D., \& Graham, J. R. 1998, ApJ, 502, 614

van Dokkum, P. G., et al. 2006, ApJ, 638, L59

Venemans, B. P., Kurk, J. D., Miley, G. K., \& Röttgering, H. J. A. 2003, NewA Rev., 47, 353

Venemans, B. P., et al. 2002, ApJ, 569, L11

.2004, A\&A, 424, L17

. 2005, A\&A, 431, 793

2007, A\&A, 461, 823

Vernet, J., Fosbury, R. A. E., Villar-Martin, M., Cohen, M. H., Cimatti, A., di Serego Alighieri, S., \& Goodrich, R. W. 2001, A\&A, 366, 7

Villar-Martín, M., et al. 2003, MNRAS, 346, 273

. 2006, MNRAS, 366, L1

Waddington, I., Windhorst, R. A., Cohen, S. H., Partridge, R. B., Spinrad, H., \& Stern, D. 1999, ApJ, 526, L77

Waddington, I., et al. 2002, MNRAS, 336, 1342

Werner, M. W., et al. 2004, ApJS, 154, 1

Willott, C. J., Rawlings, S., Blundell, K. M., \& Lacy, M. 1999, MNRAS, 309, 1017

Willott, C. J., Rawlings, S., Blundell, K. M., Lacy, M., \& Eales, S. A. 2001 MNRAS, 322, 536

Willott, C. J., Rawlings, S., Jarvis, M. J., \& Blundell, K. M. 2003, MNRAS, 339,173

Zirm, A., Dickinson, M., \& Dey, A. 2003, ApJ, 585, 90 